\title{
Periodic solutions of singular radially symmetric systems with superlinear growth
}

\author{
Alessandro Fonda • Rodica Toader • Fabio Zanolin
}

Received: 23 June 2010 / Accepted: 17 November 2010 / Published online: 16 December 2010

(C) Fondazione Annali di Matematica Pura ed Applicata and Springer-Verlag 2010

\begin{abstract}
We prove the existence of infinitely many periodic solutions for periodically forced radially symmetric systems of second-order ODE's, with a singularity of repulsive type, where the nonlinearity has a superlinear growth at infinity. These solutions have periods, which are large integer multiples of the period of the forcing, and rotate exactly once around the origin in their period time, while having a fast oscillating radial component. Analogous results hold in the case of an annular potential well.
\end{abstract}

Keywords Periodic solutions · Systems with singularity $\cdot$ Nonlinear dynamics · Continuation theorems

Mathematics Subject Classification (2000) $\quad 34 \mathrm{C} 25 \cdot 47 \mathrm{H} 15$

\section{Introduction}

We consider the system

$$
\ddot{x}=(-g(|x|)+e(t,|x|)) \frac{x}{|x|},
$$

\footnotetext{
A. Fonda $(\varangle)$

Dipartimento di Matematica e Informatica, Università di Trieste, P.le Europa 1, 34127 Trieste, Italy e-mail: a.fonda@units.it

R. Toader

Dipartimento di Ingegneria Civile e Architettura, Università di Udine, Via delle Scienze 206, 33100 Udine, Italy

e-mail: toader@uniud.it

F. Zanolin

Dipartimento di Matematica e Informatica, Università di Udine, Via delle Scienze 206,

33100 Udine, Italy

e-mail: fabio.zanolin@uniud.it
} 
where $g:] a, b[\rightarrow \mathbb{R}$ is a continuous superlinear function, with $0 \leq a<b \leq+\infty$, and $e: \mathbb{R} \times] a, b[\rightarrow \mathbb{R}$ is a Carathéodory bounded function, $T$-periodic in its first variable. Here, and in the following, $|x|$ denotes the Euclidean norm of $x \in \mathbb{R}^{N}$.

The solutions $x(t) \in \mathbb{R}^{N}$ are functions which never attain the singularity set, in the sense that

$$
|x(t)| \in] a, b[, \quad \text { for every } t \in \mathbb{R} .
$$

There is a large literature on this kind of problems. The case $a=0, b=+\infty$ has been studied, mainly by the use of variational methods, assuming that 0 is a singularity, which can be either of attractive or of repulsive type. A classical model for the attractive case is the Newton equation describing the motion of a body in a gravitational central field. Starting with Gordon [25], many attempts have been done in order to deal with periodically perturbed systems of this type, even without radial symmetry. See, e.g., $[1,8,12,37]$ and the references therein, for the variational approach, and [9,26,29,39], where topological methods were employed, instead. The repulsive case, modelling the Coulomb equation governing the motion of electrical charges, has been treated, e.g., in [10,16,26,38].

Recently, exploiting the radial symmetry of system (1), the existence of infinitely many periodic solutions was proved in [18-21], both in the attractive and in the repulsive case. The main idea there was to split the system into its radial and angular component and to consider the (scalar) angular momentum as a parameter. A rather precise description of the structure of the solution set was also obtained in [22].

In the above quoted references, having in mind, as a model, the Newton or the Coulomb equations, the nonlinearity was always assumed to be of sublinear type, or having at most a linear growth at infinity. In this situation, it is often possible to obtain some a priori bounds on the periodic solutions of the system and to recover the compactness, which is usually required in order to apply the standard variational or topological methods.

In this paper, we assume that the nonlinearity is of superlinear type. In order to explain what we mean, let us first focus on what happens at $b$. Let $G:] a, b[\rightarrow \mathbb{R}$ be a primitive of $g$, e.g.,

$$
G(r)=\int_{\bar{r}}^{r} g(\rho) \mathrm{d} \rho,
$$

where $\bar{r}$ is a fixed value in ] $a, b$. If $b=+\infty$, we will assume the standard superlinear condition

$$
\lim _{r \rightarrow+\infty} \frac{g(r)}{r}=+\infty
$$

which, by de l'Hôpital's rule, also yields

$$
\lim _{r \rightarrow+\infty} \frac{G(r)}{r^{2}}=+\infty
$$

We recall that there have been many papers dealing with scalar superlinear equations without singularities (see, e.g., [5,13,14,27,30,33]), which can be motivated by physical models involving nonlinear elasticity (see, e.g., [41]), or Bose-Einstein condensates in periodic lattices (see, e.g., [34]). In these papers, different methods have been used to detect the existence of periodic solutions, among which degree theory, the Poincaré-Birkhoff fixed point theorem and KAM theory (see also [36], and the references therein, for the use of variational methods in the framework of Hamiltonian systems). The main feature of these equations is that the 
large-amplitude solutions exhibit very fast oscillations. As observed in [17], when $b<+\infty$, in order to have the same type of fast oscillations of the solutions which come close to the singularity, conditions (4) and (5) at $b$ have to be replaced by

$$
\lim _{r \rightarrow b^{-}} g(r)=+\infty, \quad \lim _{r \rightarrow b^{-}} G(r)=+\infty .
$$

Similarly, the condition to be assumed at $a$ will be

$$
\lim _{r \rightarrow a^{+}} g(r)=-\infty, \quad \lim _{r \rightarrow a^{+}} G(r)=+\infty .
$$

Notice that, with these assumptions, we have a singularity of repulsive type at $a$, and we are dealing with a potential well. More general types of potential wells have been considered, e.g., in $[3,11,15]$, where variational methods have been used to prove the existence of periodic solutions.

A major difficulty when dealing with such kind of equations is that there is no a priori bound on the periodic solutions' set, so that more subtle arguments need to be developed. In order to overcome this lack of compactness, when dealing with the scalar equation, the method proposed in [5] and developed in [4,6,7,23] was to distinguish the solutions by their number of oscillations: as it is well known, the set of periodic solutions having a prescribed number $j$ of oscillations is indeed bounded.

In this paper, we will combine this method with a perturbation argument introduced in [20], to find infinitely many periodic solutions of (1) with a prescribed number of oscillations. In order to do this, we will need to assume that the function $e(t, r)$ is even in $t$, so to reduce the study of the radial equation to a Neumann problem, for which the topological degree will be proved to be different from zero. For the computation of the degree, different methods can be used. The one we choose here, based on the evaluation of winding numbers in the phase plane, has the advantage of being geometrically intuitive.

Let us now describe our main result, in the case $a=0$ and $b=+\infty$. The solutions we find have a planar orbit and rotate slowly around the origin. At the same time, these solutions have a fast oscillating radial component. Precisely, our solutions will be denoted by $x_{k, j}$, where $k$ is a large integer telling us that the time needed to make a complete revolution around the origin is exactly $k T$, and the integer $j$ is used to distinguish the solutions by counting the number of their radial oscillations.

Theorem 1 Let the following assumptions hold.

(H1) $\lim _{r \rightarrow 0^{+}} g(r)=-\infty$;

(H2) $\lim _{r \rightarrow 0^{+}} G(r)=+\infty$;

(H3) $\lim _{r \rightarrow+\infty} \frac{g(r)}{r}=+\infty$;

(H4) There is $\eta \in L^{1}(0, T)$ such that

$$
|e(t, r)| \leq \eta(t), \quad \text { for a.e. } t \in[0, T] \text { and every } r>0 ;
$$

(H5) The function $e(t, r)$ is even in $t$, i.e.,

$$
e(-t, r)=e(t, r), \quad \text { for a.e. } t \in \mathbb{R} \text { and every } r>0 .
$$

Let $\bar{r}$ be a fixed number in $] 0,+\infty\left[\right.$. Then, for every integer $N \geq 1$ there exists a $k_{N} \geq 1$ such that, for every integer $k \geq k_{N}$, system (1) has at least $N$ periodic solutions $x_{k, 1}(t), x_{k, 2}(t), \ldots$, $x_{k, N}(t)$, with minimal period $k T$, which make exactly one revolution around the origin in the period time $k T$. Moreover, these solutions have the following properties: 
(i) the function $\left|x_{k, j}(t)\right|$ is $T$-periodic and even;

(ii) there is an integer $J \geq 1$ such that the equality $\left|x_{k, j}(t)\right|=\bar{r}$ holds for exactly $J+j$ values of $t$ in $\left[0, \frac{T}{2}[\right.$;

(iii) there is a constant $C_{N}>0$ such that

$$
\frac{1}{C_{N}}<\left|x_{k, j}(t)\right|<C_{N}
$$

for every $t \in \mathbb{R}$, every $j=1,2, \ldots, N$, and every $k \geq k_{N}$;

(iv) if $\mu_{k, j}$ denotes the angular momentum associated with $x_{k, j}(t)$, then

$$
\lim _{k \rightarrow \infty} \mu_{k, j}=0 \text {. }
$$

The solutions found in Theorem 1 thus have a periodic radial component of the same period $T$ of the forcing term $e(t, r)$, which oscillates rapidly in the period time $T$. They remain confined in a bounded annulus and have a small angular momentum.

The proof of Theorem 1 will be given in Sect. 2. We will show in Sect. 3 how to improve the above statement in order to obtain indeed two families of solutions, distinguished by having the value $\left|x_{k, j}(0)\right|$ either smaller, or larger than $\bar{r}$ (see Theorem 2 ). We will also show that, in the general situation where $0 \leq a<b \leq+\infty$, a completely analogous result holds, as stated in Theorem 3.

\section{Proof of Theorem 1}

In this section, we provide a detailed proof of Theorem 1 . Since the system is radially symmetric, the orbits of the solutions lie on a plane (see, e.g., [18, Appendix A]), so we will assume, without loss of generality, that $N=2$.

For simplicity, we take $\bar{r}=1$.

We can assume, without loss of generality, that

$$
g(r)(r-1)>0, \quad \text { for every } r \neq 1 .
$$

Let us explain why. First of all, by (H1) and (H3), there is a $\alpha>1$ such that $g(r) \leq-1$ for every $\left.r \in] 0, \frac{1}{\alpha}\right]$, and $g(r) \geq 1$ for every $r \geq \alpha$. We can now easily construct a function $\hat{g}(r)$, which coincides with $g(r)$ on $\left.] 0, \frac{1}{\alpha}\right] \cup[\alpha,+\infty$ [, and satisfies (8). Setting $\hat{e}(t, r)=e(t, r)+\hat{g}(r)-g(r)$, we have that $\hat{g}$ and $\hat{e}$ still verify all the assumptions of Theorem 1 .

By this construction, the function $g$ can also be assumed, without loss of generality, to be continuously differentiable in a neighbourhood of the point 1 , with

$$
0<g^{\prime}(1)<\left(\frac{\pi}{T}\right)^{2} .
$$

After these modifications, by (8), the point 1 is a minimum point for $G$. We can assume, defining $G$ as in (3), with $\bar{r}=1$, that $G(1)=0$.

We may write the solutions of (1) in polar coordinates:

$$
x(t)=\rho(t)(\cos \varphi(t), \sin \varphi(t)),
$$

and (2) is satisfied if $\rho(t)>0$, for every $t$. Equation (1) is then equivalent to the system

$$
\left\{\begin{array}{l}
\ddot{\rho}-\frac{\mu^{2}}{\rho^{3}}+g(\rho)=e(t, \rho), \\
\rho^{2} \dot{\varphi}=\mu,
\end{array}\right.
$$


where $\mu$ is the (scalar) angular momentum of $x(t)$. Recall that $\mu$ is constant in time along any solution, as $\frac{\mathrm{d}}{\mathrm{d} t}\left[\rho^{2}(t) \dot{\varphi}(t)\right]=0$ for every $t$, cf. [2]. In the following, when considering a solution of (S), we will always implicitly assume that $\mu \geq 0$ and $\rho>0$. The case $\mu \leq 0$ is completely similar and would lead to a symmetric class of solutions rotating around the origin in the opposite sense.

We will look for solutions for which $\rho(t)$ is $T$-periodic. More precisely, we consider the Neumann problem

$$
\left\{\begin{array}{l}
\ddot{\rho}-\frac{\mu^{2}}{\rho^{3}}+g(\rho)=e(t, \rho), \\
\dot{\rho}(0)=\dot{\rho}\left(\frac{T}{2}\right)=0 .
\end{array}\right.
$$

By (H5), and by the periodicity of $e(t, x)$ in $t$, every solution of $\left(P_{\mu}\right)$ can be extended to the whole real line to a $T$-periodic solution, with $\rho(t)=\rho(-t)$.

The strategy of the proof is to consider $\mu$ as a parameter. We first study the case $\mu=0$, introduce a further parameter $\lambda \in[0,1]$ and consider the Neumann problem

$$
\left\{\begin{array}{l}
\ddot{\rho}+g(\rho)=\lambda e(t, \rho), \\
\dot{\rho}(0)=\dot{\rho}\left(\frac{T}{2}\right)=0 .
\end{array}\right.
$$

By the use of degree theory, we will be able to develop a double continuation argument: first, for $\mu=0$ and $\lambda$ going from 0 to 1 ; then, for $\lambda=1$ and $\mu$ moving from 0 to some small positive value.

Let us remark that exploiting the symmetry of the forcing term to find periodic solutions through the Neumann problem is a classical idea, which has been used by many authors. Recently, e.g., Llibre and Ortega have developed in [31] continuation arguments for the Neumann problem in order to obtain periodic orbits of the Sitnikov problem with a prescribed number of zeros.

Since it has to be $\rho(t)>0$, when considering the phase plane, we are led to the set

$$
\mathcal{S}=\left\{(u, v) \in \mathbb{R}^{2}: u>0\right\} .
$$

We introduce the function $\mathcal{N}: \mathcal{S} \rightarrow \mathbb{R}$, defined by

$$
\mathcal{N}(u, v)=\left(\frac{1}{u^{2}}+u^{2}+v^{2}\right)^{\frac{1}{2}} .
$$

It is easy to see that $\min \mathcal{N}=\mathcal{N}(1,0)=\sqrt{2}$.

Since we are not assuming the uniqueness of the solutions to the Cauchy problems associated with our differential equations, some care will be needed, in the following, in order to obtain uniform estimates on all the possible solutions.

Lemma 1 For every $c_{1} \geq \sqrt{2}$ there is a $c_{2}>c_{1}$ such that, if $\mathcal{N}\left(u_{0}, v_{0}\right) \leq c_{1}$, then, for every $\lambda \in[0,1]$ and $t_{0} \in\left[0, \frac{T}{2}\right]$, if $\rho_{\lambda}(t)$ is a solution of the Cauchy problem

$$
\left\{\begin{array}{l}
\ddot{\rho}+g(\rho)=\lambda e(t, \rho) \\
\rho\left(t_{0}\right)=u_{0}, \quad \dot{\rho}\left(t_{0}\right)=v_{0}
\end{array}\right.
$$

then $\rho_{\lambda}(t)$ is globally defined, and

$$
\mathcal{N}\left(\rho_{\lambda}(t), \dot{\rho}_{\lambda}(t)\right) \leq c_{2},
$$

for every $t \in\left[0, \frac{T}{2}\right]$. 
Proof Let us define the function $V: \mathcal{S} \rightarrow \mathbb{R}$ as

$$
V(u, v)=\frac{1}{2} v^{2}+G(u)+\frac{1}{2} .
$$

The solution $\rho_{\lambda}(t)$ is defined on a maximal interval $] t_{\lambda}^{-}, t_{\lambda}^{+}[$. By (H4) we have

$$
\begin{aligned}
\left|\frac{\mathrm{d}}{\mathrm{d} t} V\left(\rho_{\lambda}(t), \dot{\rho}_{\lambda}(t)\right)\right| & =\left|\dot{\rho}_{\lambda}(t) \ddot{\rho}_{\lambda}(t)+g\left(\rho_{\lambda}(t)\right) \dot{\rho}_{\lambda}(t)\right| \\
& =\left|\lambda \dot{\rho}_{\lambda}(t) e\left(t, \rho_{\lambda}(t)\right)\right| \\
& \leq \eta(t) V\left(\rho_{\lambda}(t), \dot{\rho}_{\lambda}(t)\right),
\end{aligned}
$$

for almost every $t \in] t_{\lambda}^{-}, t_{\lambda}^{+}[$. Hence, for every $t \in] t_{\lambda}^{-}, t_{\lambda}^{+}[$,

$$
V\left(\rho_{\lambda}(t), \dot{\rho}_{\lambda}(t)\right) \leq V\left(u_{0}, v_{0}\right) \exp \left|\int_{t_{0}}^{t} \eta(s) \mathrm{d} s\right| .
$$

As a consequence, we see that it has to be $] t_{\lambda}^{-}, t_{\lambda}^{+}[=]-\infty,+\infty[$.

Let $c_{1} \geq \sqrt{2}$ be given. By the properties of $\mathcal{N}$ and $V$, there is a $c_{1}^{\prime}>0$ such that, for $(u, v) \in \mathcal{S}$,

$$
\mathcal{N}(u, v) \leq c_{1} \Rightarrow V(u, v) \leq c_{1}^{\prime} .
$$

Hence, if $\mathcal{N}\left(u_{0}, v_{0}\right) \leq c_{1}$, then

$$
V\left(\rho_{\lambda}(t), \dot{\rho}_{\lambda}(t)\right) \leq c_{1}^{\prime} \exp \left(\|\eta\|_{1}\right),
$$

for every $t \in\left[0, \frac{T}{2}\right]$. Since, by (H2) and (H3),

$$
\lim _{x \rightarrow 0^{+}} G(x)=\lim _{x \rightarrow+\infty} G(x)=+\infty,
$$

there is a $c_{2}>0$ such that, for $(u, v) \in \mathcal{S}$,

$$
V(u, v) \leq c_{1}^{\prime} \exp \left(\|\eta\|_{1}\right) \Rightarrow \mathcal{N}(u, v) \leq c_{2} .
$$

It follows that, if $\mathcal{N}\left(u_{0}, v_{0}\right) \leq c_{1}$, then

$$
\mathcal{N}\left(\rho_{\lambda}(t), \dot{\rho}_{\lambda}(t)\right) \leq c_{2},
$$

for every $t \in\left[0, \frac{T}{2}\right]$, thus completing the proof.

As an immediate consequence of Lemma 1, for the Cauchy problem

$$
\left\{\begin{array}{l}
\ddot{\rho}+g(\rho)=\lambda e(t, \rho), \\
\rho(0)=u_{0}, \quad \dot{\rho}(0)=v_{0},
\end{array}\right.
$$

we have the following.

Lemma 2 For every $c_{1} \geq \sqrt{2}$ there is a $c_{2}>c_{1}$ such that, if $\mathcal{N}\left(u_{0}, v_{0}\right) \geq c_{2}$, then, for every $\lambda \in[0,1]$, if $\rho_{\lambda}\left(t ; u_{0}, v_{0}\right)$ is a solution of $\left(C P_{\lambda}\right)$, then

$$
\mathcal{N}\left(\rho_{\lambda}\left(t ; u_{0}, v_{0}\right), \dot{\rho}_{\lambda}\left(t ; u_{0}, v_{0}\right)\right) \geq c_{1},
$$

for every $t \in\left[0, \frac{T}{2}\right]$. 
By Lemma 2 , there is a $\bar{c}>\sqrt{2}$ such that, if $\mathcal{N}\left(u_{0}, v_{0}\right) \geq \bar{c}$, then, for any solution $\rho_{\lambda}\left(t ; u_{0}, v_{0}\right)$ of $\left(C P_{\lambda}\right)$,

$$
\left(\rho_{\lambda}\left(t ; u_{0}, v_{0}\right), \dot{\rho}_{\lambda}\left(t ; u_{0}, v_{0}\right)\right) \neq(1,0), \quad \text { for every } t \in\left[0, \frac{T}{2}\right] .
$$

In such a case, we can use polar coordinates

$$
\begin{aligned}
& \rho_{\lambda}\left(t ; u_{0}, v_{0}\right)=1+r_{\lambda}\left(t ; u_{0}, v_{0}\right) \cos \left(\theta_{\lambda}\left(t ; u_{0}, v_{0}\right)\right), \\
& \dot{\rho}_{\lambda}\left(t ; u_{0}, v_{0}\right)=r_{\lambda}\left(t ; u_{0}, v_{0}\right) \sin \left(\theta_{\lambda}\left(t ; u_{0}, v_{0}\right)\right) .
\end{aligned}
$$

In particular, there is a $\bar{\alpha}>1$ such that, if $\left.u_{0} \in\right] 0, \frac{1}{\bar{\alpha}}[\cup] \bar{\alpha},+\infty\left[\right.$ and $v_{0}=0$, then (11) holds.

Lemma 3 If $v_{0}=0$, we have

$$
\begin{aligned}
& \text { (a) } \lim _{u_{0} \rightarrow 0^{+}} \mathcal{N}\left(\rho_{\lambda}\left(t ; u_{0}, 0\right), \dot{\rho}_{\lambda}\left(t ; u_{0}, 0\right)\right)=\lim _{u_{0} \rightarrow+\infty} \mathcal{N}\left(\rho_{\lambda}\left(t ; u_{0}, 0\right), \dot{\rho}_{\lambda}\left(t ; u_{0}, 0\right)\right)=+\infty, \\
& \text { uniformly in } t \in\left[0, \frac{T}{2}\right] ; \\
& \text { (b) } \lim _{u_{0} \rightarrow 0^{+}} \theta_{\lambda}\left(\frac{T}{2} ; u_{0}, 0\right)=\lim _{u_{0} \rightarrow+\infty} \theta_{\lambda}\left(\frac{T}{2} ; u_{0}, 0\right)=-\infty
\end{aligned}
$$

The above limits are uniform with respect to $\lambda \in[0,1]$ and to the set of all possible solutions of the Cauchy problem $\left(C P_{\lambda}\right)$, with $v_{0}=0$.

Proof Property (a) is a direct consequence of Lemma 2. Let us prove $(b)$. Consider the problem

$$
\left\{\begin{array}{l}
\dot{u}=v+\lambda E_{u}(t), \\
\dot{v}=-g(u), \\
u(0)=u_{0}, \quad v(0)=0,
\end{array}\right.
$$

where

$$
E_{u}(t)=\int_{0}^{t} e(s, u(s)) \mathrm{d} s .
$$

We see that a solution of $\left(C P_{\lambda}^{\prime}\right)$ is given by

$$
\begin{aligned}
& u_{\lambda}\left(t ; u_{0}, 0\right)=\rho_{\lambda}\left(t ; u_{0}, 0\right), \\
& v_{\lambda}\left(t ; u_{0}, 0\right)=\dot{\rho}_{\lambda}\left(t ; u_{0}, 0\right)-\lambda \int_{0}^{t} e\left(s, \rho_{\lambda}\left(s ; u_{0}, 0\right)\right) \mathrm{d} s .
\end{aligned}
$$

Notice that $u_{\lambda}$ is the same as $\rho_{\lambda}$. By (H4), for every continuous function $u:\left[0, \frac{T}{2}\right] \rightarrow$ ] $0,+\infty[$,

$$
\left\|E_{u}\right\|_{\infty} \leq\|\eta\|_{1}
$$

so that the difference of $v_{\lambda}$ from $\dot{\rho}_{\lambda}$ is bounded. Hence, by (a),

$$
\lim _{u_{0} \rightarrow 0^{+}} \mathcal{N}\left(u_{\lambda}\left(t ; u_{0}, 0\right), v_{\lambda}\left(t ; u_{0}, 0\right)\right)=\lim _{u_{0} \rightarrow+\infty} \mathcal{N}\left(u_{\lambda}\left(t ; u_{0}, 0\right), v_{\lambda}\left(t ; u_{0}, 0\right)\right)=+\infty
$$


uniformly in $t \in\left[0, \frac{T}{2}\right]$ and $\lambda \in[0,1]$. So, there is a $\beta>1$ such that, if $\left.u_{0} \notin\right] \frac{1}{\beta}, \beta[$, the solution, which we denote by $\left(u_{\lambda}(t), v_{\lambda}(t)\right)$ for simplicity, can be written in polar coordinates

$$
u_{\lambda}(t)=1+\xi_{\lambda}(t) \cos \left(\vartheta_{\lambda}(t)\right), \quad v_{\lambda}(t)=\xi_{\lambda}(t) \sin \left(\vartheta_{\lambda}(t)\right) .
$$

We have

$$
-\dot{\vartheta}_{\lambda}=\frac{\dot{u}_{\lambda} v_{\lambda}-\dot{v}_{\lambda}\left(u_{\lambda}-1\right)}{\left(u_{\lambda}-1\right)^{2}+v_{\lambda}^{2}}=\frac{v_{\lambda}^{2}+\lambda v_{\lambda} E_{u_{\lambda}}+g\left(u_{\lambda}\right)\left(u_{\lambda}-1\right)}{\left(u_{\lambda}-1\right)^{2}+v_{\lambda}^{2}},
$$

almost everywhere in $\left[0, \frac{T}{2}\right]$. We will show that

$$
\lim _{u_{0} \rightarrow 0^{+}} \vartheta_{\lambda}\left(\frac{T}{2} ; u_{0}, 0\right)=\lim _{u_{0} \rightarrow+\infty} \vartheta_{\lambda}\left(\frac{T}{2} ; u_{0}, 0\right)=-\infty
$$

i.e., that for every $\Theta>0$ there is a $d \geq \bar{\beta}$ such that, if $\left.u_{0} \notin\right] \frac{1}{d}, d[$, then

$$
\vartheta_{\lambda}\left(\frac{T}{2} ; u_{0}, 0\right) \leq-\Theta
$$

Fix $\Theta>0$ and set

$$
C_{1}=\max \left\{\left(\frac{4(\Theta+\pi)}{T}\right)^{2}, 4\|\eta\|_{1}^{2}\right\} .
$$

We will evaluate the angular velocity outside a suitable rectangle in $\mathcal{S}$, containing $(1,0)$. The construction of such a rectangle is made in two steps.

By (H1) and (H3), there is a $d_{1} \geq 2$ such that, if $\left.u \notin\right] \frac{1}{d_{1}}, d_{1}[$, then

$$
g(u)(u-1) \geq C_{1}(u-1)^{2} .
$$

Hence, as long as $\left.u_{\lambda}(t) \notin\right] \frac{1}{d_{1}}, d_{1}\left[\right.$, since $d_{1} \geq 2$ and $C_{1} \geq 4\|\eta\|_{1}^{2}$,

$$
\begin{aligned}
-\dot{\vartheta}_{\lambda} & \geq \frac{v_{\lambda}^{2}+\lambda v_{\lambda} E_{u_{\lambda}}+C_{1}\left(u_{\lambda}-1\right)^{2}}{\left(u_{\lambda}-1\right)^{2}+v_{\lambda}^{2}} \\
& \geq \frac{v_{\lambda}^{2}-\left|v_{\lambda}\right|\|\eta\|_{1}+C_{1}\left(u_{\lambda}-1\right)^{2}}{\left(u_{\lambda}-1\right)^{2}+v_{\lambda}^{2}} \\
& \geq \frac{1}{2} \frac{v_{\lambda}^{2}+C_{1}\left(u_{\lambda}-1\right)^{2}}{\left(u_{\lambda}-1\right)^{2}+v_{\lambda}^{2}}
\end{aligned}
$$

for almost every such $t$. Let $\gamma_{1}>0$ be such that, if $u \in\left[\frac{1}{d_{1}}, d_{1}\right]$, then

$$
g(u)(u-1) \geq \frac{1}{2} C_{1}(u-1)^{2}-\gamma_{1}^{2} .
$$

Set

$$
D_{1}=2 \sqrt{\gamma_{1}^{2}+\|\eta\|_{1}^{2}}
$$


Then, as long as $u_{\lambda}(t) \in\left[\frac{1}{d_{1}}, d_{1}\right]$ and $\left|v_{\lambda}(t)\right| \geq D_{1}$, using the inequality $\left|v E_{u}\right| \leq E_{u}^{2}+\frac{1}{4} v^{2}$ and (12), we get

$$
\begin{aligned}
-\dot{\vartheta}_{\lambda} & \geq \frac{v_{\lambda}^{2}+\lambda v_{\lambda} E_{u_{\lambda}}+\frac{1}{2} C_{1}\left(u_{\lambda}-1\right)^{2}-\gamma_{1}^{2}}{\left(u_{\lambda}-1\right)^{2}+v_{\lambda}^{2}} \\
& \geq \frac{\frac{3}{4} v_{\lambda}^{2}-E_{u_{\lambda}}^{2}+\frac{1}{2} C_{1}\left(u_{\lambda}-1\right)^{2}-\gamma_{1}^{2}}{\left(u_{\lambda}-1\right)^{2}+v_{\lambda}^{2}} \\
& \geq \frac{\frac{1}{2} v_{\lambda}^{2}+\frac{1}{4} D_{1}^{2}-\|\eta\|_{1}^{2}+\frac{1}{2} C_{1}\left(u_{\lambda}-1\right)^{2}-\gamma_{1}^{2}}{\left(u_{\lambda}-1\right)^{2}+v_{\lambda}^{2}} \\
& \geq \frac{1}{2} \frac{v_{\lambda}^{2}+C_{1}\left(u_{\lambda}-1\right)^{2}}{\left(u_{\lambda}-1\right)^{2}+v_{\lambda}^{2}}
\end{aligned}
$$

for almost every such $t$. Hence, for almost every $t$, if $\left(u_{\lambda}(t), v_{\lambda}(t)\right) \notin\left[\frac{1}{d_{1}}, d_{1}\right] \times\left[-D_{1}, D_{1}\right]$, then

$$
-\dot{\vartheta}_{\lambda}(t) \geq \frac{1}{2} \frac{v_{\lambda}^{2}(t)+C_{1}\left(u_{\lambda}(t)-1\right)^{2}}{\left(u_{\lambda}(t)-1\right)^{2}+v_{\lambda}^{2}(t)}=\frac{1}{2}\left(\sin ^{2} \vartheta_{\lambda}(t)+C_{1} \cos ^{2} \vartheta_{\lambda}(t)\right) .
$$

We have thus constructed the rectangle $\mathcal{R}=\left[\frac{1}{d_{1}}, d_{1}\right] \times\left[-D_{1}, D_{1}\right]$.

Being $\mathcal{N}$ continuous, it is bounded on $\mathcal{R}$. Hence, by (13), there is a $d>0$ such that, if $\left.u_{0} \notin\right] \frac{1}{d}, d\left[\right.$, then $\left(u_{\lambda}(t), v_{\lambda}(t)\right) \notin \mathcal{R}$, for every $t \in\left[0, \frac{T}{2}\right]$. Therefore, $\dot{\vartheta}_{\lambda}\left(t ; u_{0}, 0\right)<0$ for almost every $t \in\left[0, \frac{T}{2}\right]$. Hence, since $\vartheta_{\lambda}\left(\cdot ; u_{0}, 0\right)$ is absolutely continuous, it has to be strictly decreasing.

We are now ready to evaluate $\vartheta\left(\frac{T}{2} ; u_{0}, 0\right)$, for $\left.u_{0} \notin\right] \frac{1}{d}, d[$. To fix the ideas, assume $u_{0} \geq d$. Let $t_{1}>0$ be such that $v_{\lambda}\left(t_{1}\right)=0$ and $v_{\lambda}(t)<0$ for every $\left.t \in\right] 0, t_{1}[$. Then, by the choice of $C_{1}$,

$$
t_{1} \leq \int_{0}^{-\pi} \frac{-\mathrm{d} \theta}{\frac{1}{2}\left(\sin ^{2} \theta+C_{1} \cos ^{2} \theta\right)}=\frac{2 \pi}{\sqrt{C_{1}}}<\frac{T}{2} .
$$

Analogously, let $t_{2}>t_{1}$ be such that $v_{\lambda}\left(t_{2}\right)=0$ and $v_{\lambda}(t)>0$ for every $\left.t \in\right] t_{1}, t_{2}[$. Then,

$$
t_{2}-t_{1} \leq \int_{-\pi}^{-2 \pi} \frac{-\mathrm{d} \theta}{\frac{1}{2}\left(\sin ^{2} \theta+C_{1} \cos ^{2} \theta\right)}=\frac{2 \pi}{\sqrt{C_{1}}}
$$

Continuing in this way, we find $t_{1}<t_{2}<\cdots<t_{m} \leq \frac{T}{2}<t_{m+1}$, such that $v_{\lambda}\left(t_{i}\right)=0$ and $v_{\lambda}$ has a constant sign on $] t_{i}, t_{i+1}[$, for $i=1,2, \ldots, m$. Hence,

$$
\frac{T}{2}-\frac{2 \pi}{\sqrt{C_{1}}}<t_{m} \leq m \frac{2 \pi}{\sqrt{C_{1}}}
$$

so that

$$
\vartheta_{\lambda}\left(\frac{T}{2} ; u_{0}, 0\right) \leq-m \pi<-\left(\frac{T \sqrt{C_{1}}}{4 \pi}-1\right) \pi \leq-\Theta .
$$

The same conclusion is reached, analogously, if $\left.\left.u_{0} \in\right] 0, \frac{1}{d}\right]$. We have thus proved (14). Since $\rho_{\lambda}(t)=u_{\lambda}(t)$ and $\left|\dot{\rho}_{\lambda}(t)-v_{\lambda}(t)\right| \leq\|\eta\|_{1}$, for every $t \in\left[0, \frac{T}{2}\right]$, using (14) we conclude that (b) holds, as well. 
Let us consider now the case $\lambda=0$, corresponding to the autonomous equation $\ddot{\rho}+g(\rho)$ $=0$, for which the energy $\frac{1}{2} \dot{\rho}^{2}(t)+G(\rho(t))$ remains constant in $t$. The solution $\rho_{0}\left(t ; u_{0}, v_{0}\right)$ to the Cauchy problem $\left(C P_{0}\right)$ is unique, and it is periodic. When considered in the phase plane, its orbit rotates clockwise around the point $(1,0)$. Let us denote by $\tau\left(u_{0}, v_{0}\right)$ the minimal period of $\rho_{0}\left(t ; u_{0}, v_{0}\right)$, for $\left(u_{0}, v_{0}\right) \neq(1,0)$. By Lemma 3 ,

$$
\lim _{u_{0} \rightarrow 0^{+}} \tau\left(u_{0}, 0\right)=\lim _{u_{0} \rightarrow+\infty} \tau\left(u_{0}, 0\right)=0,
$$

and, by (9),

$$
\lim _{u_{0} \rightarrow 1} \tau\left(u_{0}, 0\right)=\frac{2 \pi}{\sqrt{g^{\prime}(1)}}>2 T .
$$

For every integer $j \geq 1$, we choose $\varepsilon_{j}>0$ in such a way that

$$
2 T>T+\varepsilon_{1}>T-\varepsilon_{1}>\frac{T}{2}+\varepsilon_{2}>\frac{T}{2}-\varepsilon_{2}>\frac{T}{3}+\varepsilon_{3}>\frac{T}{3}-\varepsilon_{3}>\cdots,
$$

and consider the set

$$
\mathcal{G}_{j}=\left\{\left(u_{0}, v_{0}\right) \in \mathcal{S}: \frac{T}{j}-\varepsilon_{j}<\tau\left(u_{0}, v_{0}\right)<\frac{T}{j}+\varepsilon_{j}\right\} .
$$

By (15) and (16), since $\tau(\cdot, \cdot)$ is a continuous function, the set $\mathcal{G}_{j}$ must be nonempty, for every $j \geq 1$, and it is bounded and bounded away from the line $\left\{u_{0}=0\right\}$. It has a finite, or at most countable number of connected components, which are disjoint open annuli.

We are going to apply a duality principle from [28] in order to evaluate the topological degree associated with our problem. To this aim, we define the function $\mathcal{U}: \mathcal{S} \rightarrow \mathbb{R}^{2}$, as

$$
\mathcal{U}\left(u_{0}, v_{0}\right)=\left(u_{0}+v_{0}, v_{0}+\dot{\rho}_{0}\left(\frac{T}{2} ; u_{0}, v_{0}\right)\right) .
$$

In the following lemma, we compute the Brouwer degree of $I-\mathcal{U}$ on the set $\mathcal{G}_{j}$.

Lemma 4 For every $j \geq 1$,

$$
d_{B}\left(I-\mathcal{U}, \mathcal{G}_{j}\right)=2(-1)^{j} .
$$

Proof We first verify that the degree is well-defined. Indeed, if $(I-\mathcal{U})\left(u_{0}, v_{0}\right)=0$ and $\left(u_{0}, v_{0}\right) \in \overline{\mathcal{G}}_{j}$, then $v_{0}=0$ and $\dot{\rho}_{0}\left(\frac{T}{2} ; u_{0}, 0\right)=0$, so that the corresponding solution is $T$-periodic, and it has to be $\tau\left(u_{0}, v_{0}\right)=\frac{T}{j}$. Hence, $0 \notin(I-\mathcal{U})\left(\partial \mathcal{G}_{j}\right)$.

Let us denote the connected components of $\mathcal{G}_{j}$ by $\mathcal{G}_{j}^{1}, \mathcal{G}_{j}^{2}, \ldots$ We claim that we can find a finite number $m_{j}$ of them so that

$$
d_{B}\left(I-\mathcal{U}, \mathcal{G}_{j}\right)=d_{B}\left(I-\mathcal{U}, \mathcal{G}_{j}^{1} \cup \mathcal{G}_{j}^{2} \cup \cdots \cup \mathcal{G}_{j}^{m_{j}}\right) .
$$

Indeed, since $0 \notin(I-\mathcal{U})\left(\partial \mathcal{G}_{j}\right)$, by continuity there is a $\delta>0$ such that

$$
\operatorname{dist}\left(\left(u_{0}, v_{0}\right), \partial \mathcal{G}_{j}\right) \leq \delta \Rightarrow(I-\mathcal{U})\left(u_{0}, v_{0}\right) \neq 0 .
$$

Since $\mathcal{G}_{j}$ is bounded, there will be an integer $m_{j}$ such that, if $k>m_{j}$, then all points $\left(u_{0}, v_{0}\right) \in \mathcal{G}_{j}^{k}$ are such that $\operatorname{dist}\left(\left(u_{0}, v_{0}\right), \partial \mathcal{G}_{j}\right) \leq \delta$. The claim then follows by the excision property of the degree.

We can assume that the sets $\mathcal{G}_{j}^{1}, \mathcal{G}_{j}^{2}, \ldots, \mathcal{G}_{j}^{m_{j}}$ are ordered by increasing amplitude. Notice that

$$
d_{B}\left(I-\mathcal{U}, \mathcal{G}_{j}\right)=d_{B}\left(\mathcal{U}-I, \mathcal{G}_{j}\right)
$$


and

$$
(\mathcal{U}-I)\left(u_{0}, v_{0}\right)=\left(v_{0}, \dot{\rho}_{0}\left(\frac{T}{2} ; u_{0}, v_{0}\right)\right) .
$$

In order to compute the degree, it is convenient to introduce the function $F: \mathcal{S} \rightarrow \mathbb{R}^{2}$, defined as

$$
F\left(u_{0}, v_{0}\right)=\left(\dot{\rho}_{0}\left(\frac{T}{2} ; u_{0}, v_{0}\right), v_{0}\right)
$$

Then,

$$
d_{B}\left(\mathcal{U}-I, \mathcal{G}_{j}\right)=-d_{B}\left(F, \mathcal{G}_{j}\right) .
$$

We want to evaluate

$$
d_{B}\left(F, \mathcal{G}_{j}\right)=d_{B}\left(F, \mathcal{G}_{j}^{1}\right)+d_{B}\left(F, \mathcal{G}_{j}^{2}\right)+\cdots+d_{B}\left(F, \mathcal{G}_{j}^{m_{j}}\right) .
$$

Notice that $F=\left(F_{1}, F_{2}\right)$, with

$$
F_{1}\left(u_{0}, v_{0}\right)=\dot{\rho}_{0}\left(\frac{T}{2} ; u_{0}, v_{0}\right), \quad F_{2}\left(u_{0}, v_{0}\right)=v_{0} .
$$

Let us compute the degree $d_{B}\left(F, \mathcal{G}_{j}^{k}\right)$, for $k=1,2, \ldots, m_{j}$, evaluating the winding numbers on the outer and inner boundaries of $\mathcal{G}_{j}^{k}$.

Assume that $\tau$ equals $\frac{T}{j}+\varepsilon_{j}$ on the inner boundary, and $\frac{T}{j}-\varepsilon_{j}$ on the outer boundary of $\mathcal{G}_{j}^{k}$. To compute the winding number, let us start by taking the point $\left(u_{0}, v_{0}\right)$ equal to $\left(\breve{u}_{j}^{k}, 0\right)$ on the outer boundary, with $\breve{u}_{j}^{k}>1$. Here, $\tau\left(\breve{u}_{j}^{k}, 0\right)=\frac{T}{j}-\varepsilon_{j}$, so that, in the time $T$, the solution vector $\left(\rho_{0}\left(t ; \breve{u}_{j}^{k}, 0\right), \dot{\rho}_{0}\left(t ; \breve{u}_{j}^{k}, 0\right)\right)$ rotates around the point $(1,0)$ more than $j$ times, and less than $j+1$ times. Since, by symmetry, the time to make half a rotation is exactly half of the time needed to perform a complete rotation, we have that, in the time $\frac{T}{2}$, the solution vector makes more than $j$ half-rotations, and less that $j+1$ half-rotations.

Assume first that $j$ is odd. Then, $F_{1}\left(\breve{u}_{j}^{k}, 0\right)>0$, so that $F\left(\breve{u}_{j}^{k}, 0\right)$ lies on the half-line ] $0,+\infty[\times\{0\}$. Since $F$ does not change the second coordinate, while going around the outer boundary of $\mathcal{G}_{j}^{k}$ in clockwise direction with the point $\left(u_{0}, v_{0}\right)$, the vector $F\left(u_{0}, v_{0}\right)$ will lie in the lower half-plane, until the point $\left(u_{0}, v_{0}\right)$ reaches the point $\left(\tilde{u}_{j}^{k}, 0\right)$, with $\tilde{u}_{j}^{k} \in$ ]0, 1[, such that $G\left(\tilde{u}_{j}^{k}\right)=G\left(\tilde{u}_{j}^{k}\right)$. Here, $F_{1}\left(\tilde{u}_{j}^{k}, 0\right)<0$, so that $F\left(\tilde{u}_{j}^{k}, 0\right)$ lies on the half-line ]$-\infty, 0\left[\times\{0\}\right.$. Continuing in this way, we see that, as $\left(u_{0}, v_{0}\right)$ performs a clockwise rotation around the outer boundary of $\mathcal{G}_{j}^{k}$, the vector $F\left(u_{0}, v_{0}\right)$ also makes a single rotation, and in clockwise direction, too. So, if $j$ is odd, we see that the winding number corresponding to the outer boundary of $\mathcal{G}_{j}^{k}$ is +1 .

On the contrary, if $j$ is even, then $F_{1}\left(\check{u}_{j}^{k}, 0\right)<0$, and $F\left(\check{u}_{j}^{k}, 0\right)$ lies on the half-line ] $-\infty$, $0\left[\times\{0\}\right.$. So, the opposite situation is encountered: this time the vector $F\left(u_{0}, v_{0}\right)$ will make a single rotation, but in counter-clockwise direction, and the winding number is -1 .

Let us consider now the inner boundary. If we start from the point $\left(\hat{u}_{j}^{k}, 0\right)$ on the inner boundary, with $\hat{u}_{j}^{k}>1$, we have $\tau\left(\hat{u}_{j}^{k}, 0\right)=\frac{T}{j}+\varepsilon_{j}$. This means that, in the time $\frac{T}{2}$, the solution makes more than $j-1$ half-rotations, and less that $j$ half-rotations. We are in the opposite situation with respect to what happens on the outer boundary, so we can conclude that the winding number is -1 if $j$ is odd, and +1 if $j$ is even.

Combining this information, and using the additivity property of the degree, we conclude that, if $\tau$ equals $\frac{T}{j}+\varepsilon_{j}$ on the inner boundary, and $\frac{T}{j}-\varepsilon_{j}$ on the outer boundary of $\mathcal{G}_{j}^{k}$, then

$$
d_{B}\left(F, \mathcal{G}_{j}^{k}\right)= \begin{cases}+2 & \text { if } j \text { is odd }, \\ -2 & \text { if } j \text { is even } .\end{cases}
$$


A similar argument shows that, if $\tau$ equals $\frac{T}{j}-\varepsilon_{j}$ on the inner boundary, and $\frac{T}{j}+\varepsilon_{j}$ on the outer boundary of $\mathcal{G}_{j}^{k}$, then

$$
d_{B}\left(F, \mathcal{G}_{j}^{k}\right)= \begin{cases}-2 & \text { if } j \text { is odd, } \\ +2 & \text { if } j \text { is even. }\end{cases}
$$

Analogously, one sees that, if $\tau$ has the same values on the inner and outer boundaries, then

$$
d_{B}\left(F, \mathcal{G}_{j}^{k}\right)=0 .
$$

So, without loss of generality, for the computation of the degree, we can assume that on each annulus $\mathcal{G}_{j}^{k}$ the values of $\tau$ at the inner and outer boundaries are different. Assuming this, we now claim that, for $j$ large enough, the number $m_{j}$ is odd.

Let us start taking $u_{0}$ near the point 1 . Then, by $(16), \tau\left(u_{0}, 0\right)$ is larger than $2 T$. Letting $u_{0}$ increase, when we meet the first annulus $\mathcal{G}_{j}^{1}$, the values of $\tau$ at the inner and outer boundaries must be $\frac{T}{j}+\varepsilon_{j}$ and $\frac{T}{j}-\varepsilon_{j}$, respectively. There will be a "first instant" $\hat{u}_{j}^{1}$ when $\tau\left(\hat{u}_{j}^{1}, 0\right)=\frac{T}{j}+\varepsilon_{j}$ and $\tau\left(u_{0}, 0\right)<\frac{T}{j}+\varepsilon_{j}$ for all $u_{0}$ in a small right-neighbourhood of $\hat{u}_{j}^{1}$. For those $u_{0}$, we have that the point $\left(u_{0}, 0\right)$ is in $\mathcal{G}_{j}^{1}$. We will say that $\hat{u}_{0}^{1}$ is the "entrance point" in $\mathcal{G}_{j}^{1}$. Continuing in letting $u_{0}$ increase, we will remain inside $\mathcal{G}_{j}^{1}$ for a while, and eventually go out at some "exit point" $\check{u}_{j}^{1}$ after, where $\tau\left(\check{u}_{j}^{1}\right)=\frac{T}{j}-\varepsilon_{j}$.

It could then happen that the function $\tau$ remains below the value $\frac{T}{j}-\varepsilon_{j}$, for every $u_{0}>\hat{u}_{0}^{1}$. In this case, $m_{j}=1$, and we have proved the claim. Assume now $m_{j} \geq 2$.

The opposite situation is encountered for $\mathcal{G}_{j}^{2}$ : there is an entrance point at which the value of $\tau$ is $\frac{T}{j}-\varepsilon_{j}$, and an exit point at which the value of $\tau$ is $\frac{T}{j}+\varepsilon_{j}$. So, by (15), it cannot be that $m_{j}=2$. It has to be $m_{j} \geq 3$.

This alternating behaviour is repeated for every $\mathcal{G}_{j}^{k}$. In particular, the value of $\tau$ at the exit point is $\frac{T}{j}-\varepsilon_{j}$ if $k$ is odd, and $\frac{T}{j}+\varepsilon_{j}$ if $k$ is even. But, by (15), at the exit point of $\mathcal{G}_{j}^{m_{j}}$ the value of $\tau$ must be $\frac{T}{j}-\varepsilon_{j}$. Hence, $m_{j}$ has to be odd.

Consequently,

$$
d_{B}\left(F, \mathcal{G}_{j}\right)=\sum_{k=1}^{m_{j}} d_{B}\left(F, \mathcal{G}_{j}^{k}\right)=2(-1)^{j+1} .
$$

Combining this with (19), we have the conclusion.

Let $X=C^{1}\left(\left[0, \frac{T}{2}\right]\right)$, and set

$$
X_{+}=\{\rho \in X: \min \rho>0\} .
$$

Define the linear operator

$$
\begin{aligned}
& L: D(L) \subset X \rightarrow L^{1}(0, T), \\
& D(L)=\left\{\rho \in W^{2,1}(0, T): \dot{\rho}(0)=\dot{\rho}\left(\frac{T}{2}\right)=0\right\}, \\
& L \rho=\ddot{\rho},
\end{aligned}
$$

and the Nemytzkii operator

$$
\begin{aligned}
& \hat{N}: X_{+} \times[0,1] \rightarrow L^{1}(0, T), \\
& \hat{N}(\rho, \lambda)(t)=-g(\rho(t))+\lambda e(t, \rho(t)) .
\end{aligned}
$$


Taking $\sigma \in \mathbb{R}$ not belonging to the spectrum of $L$, we have that $\left(\hat{P}_{\lambda}\right)$ can be translated to the fixed point problem

$$
\rho=(L-\sigma I)^{-1}(\hat{N}(\cdot, \lambda)-\sigma I) \rho .
$$

Define the set

$$
\Omega_{j}=\left\{\rho \in X_{+}:(\rho(t), \dot{\rho}(t)) \in \mathcal{G}_{j}, \text { for every } t \in\left[0, \frac{T}{2}\right]\right\} .
$$

This is an open and bounded set in $X$, and its closure is contained in $X_{+}$. We want to compute the Leray-Schauder degree $d_{L S}$ of $I-(L-\sigma I)^{-1}(\hat{N}(\cdot, 0)-\sigma I)$ on the set $\Omega_{j}$. We will use the notation

$$
D_{L}\left(L-\hat{N}(\cdot, 0), \Omega_{j}\right)=d_{L S}\left(I-(L-\sigma I)^{-1}(\hat{N}(\cdot, 0)-\sigma I), \Omega_{j}\right),
$$

coming from the coincidence degree theory, cf. [24].

Lemma 5 For every $j \geq 1$,

$$
D_{L}\left(L-\hat{N}(\cdot, 0), \Omega_{j}\right)=2(-1)^{j} .
$$

Proof It is readily seen that the sets $\Omega_{j}$ and $\mathcal{G}_{j}$ have a common core with respect to the Neumann problem $\left(\hat{P}_{0}\right)$, cf. [28]. This means that

(i) $L \rho \neq \hat{N}(\rho, 0)$, for every $\rho \in \partial \Omega_{j}$;

(ii) $\mathcal{U}\left(u_{0}, v_{0}\right) \neq\left(u_{0}, v_{0}\right)$ for every $\left(u_{0}, v_{0}\right) \in \partial \mathcal{G}_{j}$;

(iii) the set of those $\left(u_{0}, v_{0}\right) \in \mathcal{G}_{j}$ such that $\mathcal{U}\left(u_{0}, v_{0}\right)=\left(u_{0}, v_{0}\right)$ coincides with the set initial values $(\rho(0), \dot{\rho}(0))$ of the functions $\rho \in \Omega_{j}$ which are solutions of $L \rho=\hat{N}(\rho, 0)$.

Hence, using [28, Theorem 29.4],

$$
D_{L}\left(L-\hat{N}(\cdot, 0), \Omega_{j}\right)=d_{B}\left(I-\mathcal{U}, \mathcal{G}_{j}\right) .
$$

The conclusion follows, by Lemma 4.

We now use the approach introduced in [5] and developed in [4,6,7]. Let us define the function $\kappa: X_{+} \times[0,1] \rightarrow \mathbb{R}$ as

$$
\kappa(\rho, \lambda)=\frac{1}{\pi} \int_{0}^{T / 2} \frac{\dot{\rho}^{2}(t)+(g(\rho(t))-\lambda e(t, \rho(t)))(\rho(t)-1)}{\max \left\{(\rho(t)-1)^{2}+\dot{\rho}^{2}(t), \frac{1}{4}\right\}} \mathrm{d} t .
$$

It is a continuous function. By the properties of $\mathcal{N}$, there is a constant $d_{1}>\sqrt{2}$ such that

$$
\mathcal{N}(u, v) \geq d_{1} \Rightarrow(u-1)^{2}+v^{2} \geq \frac{1}{4} .
$$

By Lemma 2, there is a $d_{2}>d_{1}$ such that, if $\rho_{\lambda} \in X_{+}$is a solution of $\left(C P_{\lambda}\right)$, with $\lambda \in[0,1]$ and $\mathcal{N}\left(u_{0}, v_{0}\right) \geq d_{2}$, then $\mathcal{N}\left(\rho_{\lambda}(t), \dot{\rho}_{\lambda}(t)\right) \geq d_{1}$, hence $\left(\rho_{\lambda}(t)-1\right)^{2}+\dot{\rho}_{\lambda}^{2}(t) \geq \frac{1}{4}$, for every $t \in\left[0, \frac{T}{2}\right]$, so that

$$
\begin{aligned}
\kappa\left(\rho_{\lambda}, \lambda\right) & =\frac{1}{\pi} \int_{0}^{T / 2} \frac{\dot{\rho}_{\lambda}^{2}(t)+\left(g\left(\rho_{\lambda}(t)\right)-\lambda e\left(t, \rho_{\lambda}(t)\right)\right)\left(\rho_{\lambda}(t)-1\right)}{\left(\rho_{\lambda}(t)-1\right)^{2}+\dot{\rho}_{\lambda}^{2}(t)} \mathrm{d} t \\
& =\frac{1}{\pi}\left(\theta_{\lambda}(0)-\theta_{\lambda}\left(\frac{T}{2}\right)\right) .
\end{aligned}
$$


By the definition of the function $\mathcal{N}$, the following two implications are easy to verify:

$$
u \notin] \frac{1}{d_{2}}, d_{2}\left[\Rightarrow \mathcal{N}(u, 0) \geq d_{2},\right.
$$

and

$$
\mathcal{N}(u, 0)>d_{2} \sqrt{2} \Rightarrow u \notin\left[\frac{1}{d_{2}}, d_{2}\right] .
$$

As a consequence of (20), if $\rho_{\lambda}$ solves the Neumann problem $\left(\hat{P}_{\lambda}\right)$, with

$$
\left.\rho_{\lambda}(0) \notin\right] \frac{1}{d_{2}}, d_{2}[,
$$

then $\kappa\left(\rho_{\lambda}, \lambda\right)$ counts the number of half-rotations around $(1,0)$ of $\left(\rho_{\lambda}(t), \dot{\rho}_{\lambda}(t)\right)$, in the phase plane, as $t$ varies from 0 to $\frac{T}{2}$.

Lemma 6 There is a $\kappa_{1}>0$ such that, if $\rho_{\lambda} \in X_{+}$is a solution of $\left(C P_{\lambda}\right)$ with $\lambda \in[0,1]$ and $\mathcal{N}\left(u_{0}, v_{0}\right) \leq d_{2} \sqrt{2}$, then $\kappa\left(\rho_{\lambda}, \lambda\right) \leq \kappa_{1}$.

Proof By Lemma 1, there is a $d_{2}^{\prime}>d_{2}$ such that, if $\mathcal{N}\left(u_{0}, v_{0}\right) \leq d_{2} \sqrt{2}$, then $\mathcal{N}\left(\rho_{\lambda}(t), \dot{\rho}_{\lambda}(t)\right) \leq d_{2}^{\prime}$, for every $t \in\left[0, \frac{T}{2}\right]$. By the Carathéodory conditions, there is a $\ell \in L^{1}\left(0, \frac{T}{2}\right)$ such that

$$
\left|\frac{\dot{\rho}_{\lambda}^{2}(t)+\left(g\left(\rho_{\lambda}(t)\right)-\lambda e\left(t, \rho_{\lambda}(t)\right)\right)\left(\rho_{\lambda}(t)-1\right)}{\max \left\{\left(\rho_{\lambda}(t)-1\right)^{2}+\dot{\rho}_{\lambda}^{2}(t), \frac{1}{4}\right\}}\right| \leq \ell(t),
$$

for almost every $t \in\left[0, \frac{T}{2}\right]$. Taking $\kappa_{1}=\frac{1}{\pi}\|\ell\|_{1}$, we have the conclusion.

Lemma 7 There is a constant $C_{j}>0$ such that, if $\rho_{\lambda}$ solves the Neumann problem $\left(\hat{P}_{\lambda}\right)$, with $\lambda \in[0,1]$ and $\kappa\left(\rho_{\lambda}, \lambda\right)=j$, then

$$
\mathcal{N}\left(\rho_{\lambda}(t), \dot{\rho}_{\lambda}(t)\right)<C_{j}, \quad \text { for every } t \in\left[0, \frac{T}{2}\right] .
$$

Proof By Lemma 3, there is a $c_{j}>0$ such that, if $\left.\rho_{\lambda}(0)=u_{0} \notin\right] \frac{1}{c_{j}}, c_{j}\left[\right.$ and $\dot{\rho}_{\lambda}(0)=$ $v_{0}=0$, then the solution makes more than $j$ rotations around $(1,0)$, in the phase plane, as $t$ varies from 0 to $\frac{T}{2}$. On the other hand, by Lemma 1 , there is a $C_{j}>0$ such that, if $\mathcal{N}\left(\rho_{\lambda}\left(t_{0}\right), \dot{\rho}_{\lambda}\left(t_{0}\right)\right) \geq C_{j}$ for some $t_{0} \in\left[0, \frac{T}{2}\right]$, then $\mathcal{N}\left(\rho_{\lambda}(0), \dot{\rho}_{\lambda}(0)\right) \geq c_{j} \sqrt{2}$. Taking into account that $\dot{\rho}_{\lambda}(0)=0$, we have, as in $(21)$, that $\left.\rho_{\lambda}(0) \notin\right] \frac{1}{c_{j}}, c_{j}[$, hence the conclusion.

Define the set

$$
\begin{gathered}
\mathcal{O}_{j}=\left\{(\rho, \lambda) \in X_{+} \times[0,1]: \kappa(\rho, \lambda) \in\right] j-\frac{1}{4}, j+\frac{1}{4}\left[, \rho(0) \notin\left[\frac{1}{d_{2}}, d_{2}\right],\right. \\
\text { and } \left.\mathcal{N}(\rho(t), \dot{\rho}(t))<C_{j}, \text { for every } t \in\left[0, \frac{T}{2}\right]\right\} .
\end{gathered}
$$

It is open and bounded in $X \times[0,1]$. Let us denote by $\overline{\mathcal{O}}_{j}$ and $\partial \mathcal{O}_{j}$ the closure and the boundary of $\mathcal{O}_{j}$ in $X \times[0,1]$. Notice that $\overline{\mathcal{O}}_{j} \subset X_{+} \times[0,1]$.

After noticing that

$$
\bar{\tau}:=\inf \left\{\tau\left(u_{0}, 0\right): u_{0} \in\left[\frac{1}{d_{2}}, 1[\cup] 1, d_{2}\right]\right\}>0,
$$

let us fix an integer $J>\max \left\{\kappa_{1}, T / \bar{\tau}\right\}$, where $\kappa_{1}$ is given by Lemma 6 . 
Lemma 8 If $j \geq J$ and $\rho_{\lambda}$ solves the Neumann problem $\left(\hat{P}_{\lambda}\right)$, with $\lambda \in[0,1]$ and $\left(\rho_{\lambda}, \lambda\right) \in$ $\overline{\mathcal{O}}_{j}$, then $\left(\rho_{\lambda}, \lambda\right) \in \mathcal{O}_{j}$.

Proof If $\left(\rho_{\lambda}, \lambda\right) \in \overline{\mathcal{O}}_{j}$, we have that $\left.\kappa\left(\rho_{\lambda}, \lambda\right) \in\left[j-\frac{1}{4}, j+\frac{1}{4}\right], \rho_{\lambda}(0) \notin\right] \frac{1}{d_{2}}, d_{2}[$, and $\mathcal{N}\left(\rho_{\lambda}(t), \dot{\rho}_{\lambda}(t)\right) \leq C_{j}$, for every $t \in\left[0, \frac{T}{2}\right]$. Being $\rho_{\lambda}$ a solution of $\left(\hat{P}_{\lambda}\right)$, by the choice of $d_{2}$ we know that $\kappa\left(\rho_{\lambda}, \lambda\right)$ is an integer which counts the number of half-rotations around $(1,0)$ of $\left(\rho_{\lambda}(t), \dot{\rho}_{\lambda}(t)\right)$, in the phase plane, as $t$ varies from 0 to $\frac{T}{2}$. Hence, it has to be $\kappa\left(\rho_{\lambda}, \lambda\right)=j$. Therefore, by Lemma $7, \mathcal{N}\left(\rho_{\lambda}(t), \dot{\rho}_{\lambda}(t)\right)<C_{j}$, for every $t \in\left[0, \frac{T}{2}\right]$. Since $j>\kappa_{1}$, by Lemma 6, it has to be $\mathcal{N}\left(\rho_{\lambda}(0), \dot{\rho}_{\lambda}(0)\right)>d_{2} \sqrt{2}$, hence, since $\dot{\rho}_{\lambda}(0)=0$, by (21) we have that $\rho_{\lambda}(0) \notin\left[\frac{1}{d_{2}}, d_{2}\right]$. We have thus proved that $\left(\rho_{\lambda}, \lambda\right) \in \mathcal{O}_{j}$.

We have thus shown that $0 \notin\left(I-(L-\sigma I)^{-1}(\hat{N}-\sigma I)\right)\left(\partial \mathcal{O}_{j}\right)$, for every $j \geq J$. By a classical result in the Leray-Schauder degree theory, cf. [35, Lemma 1.8], denoting by $\mathcal{O}_{j}^{\lambda}$ the set of those $\rho$ such that $(\rho, \lambda) \in \mathcal{O}_{j}$, we have that $D_{L}\left(L-\hat{N}(\cdot, \lambda), \mathcal{O}_{j}^{\lambda}\right)$ is independent of $\lambda \in[0,1]$. Hence,

$$
D_{L}\left(L-\hat{N}(\cdot, 1), \mathcal{O}_{j}^{1}\right)=D_{L}\left(L-\hat{N}(\cdot, 0), \mathcal{O}_{j}^{0}\right) .
$$

Lemma 9 For every $j \geq J$,

$$
D_{L}\left(L-\hat{N}(\cdot, 0), \mathcal{O}_{j}^{0}\right)=2(-1)^{j} .
$$

Proof We claim that, if $\rho$ is a solution of $\left(\hat{P}_{0}\right)$, then, for $j \geq J$,

$$
\rho \in \mathcal{O}_{j}^{0} \Leftrightarrow \rho \in \Omega_{j} \text {. }
$$

Indeed, if $\rho \in \mathcal{O}_{j}^{0}$, since it solves the Neumann problem, it has to be $\kappa(\rho, 0)=j$. Recall that a solution of $\left(\hat{P}_{0}\right)$ can be naturally extended to a $T$-periodic and even function. Hence, extending $\rho$ in such a way, it has minimal period $\frac{T}{j}$ so that the orbit $(\rho(t), \dot{\rho}(t))$ belongs to $\mathcal{G}_{j}$, for every $t$. This means that $\rho \in \Omega_{j}$.

On the other hand, if $\rho \in \Omega_{j}$, then the periodic extension of $\rho$ has minimal period $\frac{T}{j}$. Since $j \geq J>T / \bar{\tau}$, we have that $\rho(0) \notin\left[\frac{1}{d_{2}}, d_{2}\right]$, hence $(\rho(t)-1)^{2}+\dot{\rho}^{2}(t)>\frac{1}{4}$ for every $t$, so that it has to be $\kappa(\rho, 0)=j$. By Lemma $7, \mathcal{N}\left(\rho_{\lambda}(t), \dot{\rho}_{\lambda}(t)\right)<C_{j}$, for every $t \in\left[0, \frac{T}{2}\right]$. So, $\rho \in \mathcal{O}_{j}^{0}$.

Having proved this, we then have that

$$
D_{L}\left(L-\hat{N}(\cdot, 0), \mathcal{O}_{j}^{0}\right)=D_{L}\left(L-\hat{N}(\cdot, 0), \Omega_{j}\right) .
$$

The conclusion follows, by Lemma 5 .

So, in particular, we have that

$$
D_{L}\left(L-\hat{N}(\cdot, 1), \mathcal{O}_{j}^{1}\right) \neq 0 .
$$

We now follow the argument in [20, Theorem 2]. Let

$$
\begin{aligned}
& N_{\mu}: X_{+} \rightarrow L^{1}(0, T), \\
& \left(N_{\mu} \rho\right)(t)=\frac{\mu^{2}}{\rho^{3}(t)}-g(\rho(t))+e(t, \rho(t)) .
\end{aligned}
$$


Problem $\left(P_{\mu}\right)$ is then equivalent to

$$
L \rho=N_{\mu} \rho .
$$

Taking $\sigma \in \mathbb{R}$ not belonging to the spectrum of $L$, we have that (24) can be translated to the fixed point problem

$$
\rho=(L-\sigma I)^{-1}\left(N_{\mu}-\sigma I\right) \rho .
$$

Since $\mathcal{O}_{j}^{1}$ is uniformly positively bounded below, the closure of $\mathcal{O}_{j}^{1}$ is contained in $X_{+}$. We first prove that there exists a constant $M_{j}>0$ such that for every $\mu \in\left[0, M_{j}\right]$ there is no solution of (24) on the boundary $\partial \mathcal{O}_{j}^{1}$. Indeed, by contradiction, assume there are two sequences $\left(\mu_{n}\right)_{n}$ and $\left(\rho_{n}\right)_{n}$ such that $\mu_{n} \rightarrow 0, \rho_{n} \in \partial \mathcal{O}_{j}^{1}$, and

$$
\rho_{n}=(L-\sigma I)^{-1}\left(N_{\mu_{n}}-\sigma I\right) \rho_{n} .
$$

Then, $\left(\rho_{n}\right)_{n}$ and $\left(1 / \rho_{n}\right)_{n}$ are uniformly bounded, so that $\left(\left(N_{\mu_{n}}-\sigma I\right) \rho_{n}\right)_{n}$ is bounded in $L^{1}(0, T)$. Being $(L-\sigma I)^{-1}: L^{1}(0, T) \rightarrow X$ a compact operator, there exists a subsequence, still denoted by $\left(\rho_{n}\right)_{n}$, for which $(L-\sigma I)^{-1}\left(N_{\mu_{n}}-\sigma I\right) \rho_{n}$ converges to some $\bar{\rho} \in X$. Hence $\rho_{n} \rightarrow \bar{\rho}$, as well, and, being $\partial \mathcal{O}_{j}^{1}$ closed, $\bar{\rho} \in \partial \mathcal{O}_{j}^{1}$. Since $\rho_{n} \rightarrow \bar{\rho}$ uniformly, and $\bar{\rho} \in X_{+}$, we deduce from the definition of $N_{\mu}$ that $\bar{\rho}=(L-\sigma I)^{-1}\left(N_{0}-\sigma I\right) \bar{\rho}$, so that $\bar{\rho}$ solves (24) with $\mu=0$, a contradiction with the assumptions.

By the global continuation principle of Leray-Schauder (see e.g. [40, Theorem 14.C], or [32, Theorem 2.2]), for every $j \geq J$, there is a continuum $\mathcal{C}_{j}$ in $\left[0, M_{j}\right] \times \mathcal{O}_{j}^{1}$ connecting $\{0\} \times \mathcal{O}_{j}^{1}$ with $\left\{M_{j}\right\} \times \mathcal{O}_{j}^{1}$, whose elements $(\mu, \rho)$ satisfy $\left(P_{\mu}\right)$. We now extend $\rho(t)$ on $\left[-\frac{T}{2}, \frac{T}{2}\right]$ as an even function, with $\rho(-t)=\rho(t)$, and then extend it further to the whole real line, by $T$-periodicity. Then, the first equation in (S) is satisfied, for all $t \in \mathbb{R}$. Let us consider the function $\Phi: \mathcal{C}_{j} \rightarrow \mathbb{R}$, defined by

$$
\Phi(\mu, \rho)=\int_{0}^{T} \frac{\mu}{\rho^{2}(t)} \mathrm{d} t .
$$

It is continuous and defined on a compact and connected domain, so its image is a compact interval. Since $\Phi(0, \rho)=0$, and $\Phi$ is not identically zero, this interval is of the type $\left[0, \bar{\theta}_{j}\right]$, for some $\bar{\theta}_{j}>0$.

Given $\theta \in\left[0, \bar{\theta}_{j}\right]$, there are $(\mu, \rho) \in \mathcal{C}_{j}$ such that

$$
\int_{0}^{T} \frac{\mu}{\rho^{2}(t)} \mathrm{d} t=\theta
$$

Defining

$$
\varphi(t)=\int_{0}^{t} \frac{\mu}{\rho^{2}(s)} \mathrm{d} s,
$$

the second equation in (S) is also satisfied and

$$
\varphi(t+T)-\varphi(t)=\int_{t}^{t+T} \frac{\mu}{\rho^{2}(s)} \mathrm{d} s=\int_{0}^{T} \frac{\mu}{\rho^{2}(s)} \mathrm{d} s=\theta,
$$


for every $t \in \mathbb{R}$. Then, for every $\theta \in\left[0, \bar{\theta}_{j}\right]$, the solution of system (S) found above provides, through (10), a solution to system (1) such that

$$
x(t+T)=e^{i \theta} x(t),
$$

for every $t \in \mathbb{R}$ (for briefness we used here the complex notation).

In particular, if $\theta=\frac{2 \pi}{k}$ for some integer $k \geq 1$, then $x(t)$ is periodic with minimal period $k T$, and rotates exactly once around the origin in the period time $k T$. Hence, for every $j \geq J$ and every integer $k \geq 2 \pi / \bar{\theta}_{j}$, we have such a $k T$-periodic solution, which we denote by $x_{k, j}(t)$. Let $\left(\rho_{k, j}(t), \varphi_{k, j}(t)\right)$ be its polar coordinates, and $\mu_{k, j}$ be its angular momentum. By the above construction, $\left(\mu_{k, j}, \rho_{k, j}, \varphi_{k, j}\right)$ verify system $(\mathrm{S}),\left(\mu_{k, j}, \rho_{k, j}\right) \in \mathcal{C}_{j}$, and

$$
\int_{0}^{T} \frac{\mu_{k, j}}{\rho_{k, j}^{2}(t)} \mathrm{d} t=\frac{2 \pi}{k} .
$$

Since $\rho_{k, j} \in \mathcal{O}_{j}^{1}$, and $\mathcal{O}_{j}^{1}$ is bounded in $C([0, T])$ and uniformly bounded below, there is a constant $c_{j}>0$ such that

$$
\frac{1}{c_{j}} \leq \rho_{k, j}(t) \leq c_{j},
$$

for every $t \in[0, T]$. Hence,

$$
\frac{2 \pi}{k}=\int_{0}^{T} \frac{\mu_{k, j}}{\rho_{k, j}^{2}(t)} \mathrm{d} t \geq T \frac{\mu_{k, j}}{c_{j}^{2}},
$$

so that $\lim _{k} \mu_{k, j}=0$.

Given a positive integer $N$, taking

$$
\theta_{N}=\min \left\{\bar{\theta}_{j}: j=J+1, J+2, \ldots, J+N\right\},
$$

for every integer $k \geq 2 \pi / \theta_{N}$ there will be at least $N$ periodic solutions

$$
x_{k, J+1}, x_{k, J+2}, \ldots, x_{k, J+N},
$$

of minimal period $k T$, which rotate exactly once around the origin in their period time. Setting

$$
C_{N}=\max \left\{c_{j}: j=J+1, J+2, \ldots, J+N\right\},
$$

the proof is readily completed, after relabelling the indices of $x_{k, j}$.

Remark 1 The solutions satisfying (25) are quasi-periodic with $T$-periodic radial component, and mean angular velocity, on every time interval of length $T$, equal to $\theta / T$.

\section{Refinements of Theorem 1}

In this section, we will show how to improve the information on the topological degree, in order to obtain the existence of $t w o$ distinct families of solutions with the properties listed in the statement of Theorem 1. We will prove the following result. 
Theorem 2 Let the assumptions (H1)-(H5) hold. Let $\bar{r}$ be a fixed number in $] 0,+\infty[$. Then, for every integer $N \geq 1$ there exists a $k_{N} \geq 1$ such that, for every integer $k \geq k_{N}$, system (1) has at least $2 N$ periodic solutions $x_{k, 1}^{(i)}(t), x_{k, 2}^{(i)}(t), \ldots, x_{k, N}^{(i)}(t)$, with $i \in\{1,2\}$, having minimal period $k T$, which make exactly one revolution around the origin in the period time $k T$. These solutions have the properties (i)-(iv) stated in Theorem 1 and, moreover,

$$
\left|x_{k, j}^{(1)}(0)\right|<\bar{r}, \quad\left|x_{k, j}^{(2)}(0)\right|>\bar{r} .
$$

Proof As in the previous section, we assume $\bar{r}=1$, and we introduce the sets $\mathcal{G}_{j}$, as in (17). Recall that they are the finite or at most countable union of open annuli in $\mathbb{R}^{2}$. As seen in Lemma 5 , for every $j \geq 1$, setting

$$
\Omega_{j}=\left\{\rho \in X_{+}:(\rho(t), \dot{\rho}(t)) \in \mathcal{G}_{j}, \text { for every } t \in\left[0, \frac{T}{2}\right]\right\},
$$

we have

$$
D_{L}\left(L-\hat{N}(\cdot, 0), \Omega_{j}\right)=2(-1)^{j} .
$$

We now define the sets

$$
\Omega_{j}^{-}=\left\{\rho \in \Omega_{j}: \rho(0)<1\right\}, \quad \Omega_{j}^{+}=\left\{\rho \in \Omega_{j}: \rho(0)>1\right\} .
$$

Since there are no solutions of $\left(\hat{P}_{0}\right)$ in $\Omega_{j}$ with $\rho(0)=1$, we have that

$$
D_{L}\left(L-\hat{N}(\cdot, 0), \Omega_{j}\right)=D_{L}\left(L-\hat{N}(\cdot, 0), \Omega_{j}^{-}\right)+D_{L}\left(L-\hat{N}(\cdot, 0), \Omega_{j}^{+}\right) .
$$

We will now show that these two last degrees have the same value.

Lemma 10 For every $j \geq 1$,

$$
D_{L}\left(L-\hat{N}(\cdot, 0), \Omega_{j}^{-}\right)=D_{L}\left(L-\hat{N}(\cdot, 0), \Omega_{j}^{+}\right)=(-1)^{j} .
$$

Proof Let us denote by $\mathcal{P}_{1} \mathcal{G}_{j}$ the projections of the sets $\mathcal{G}_{j}$ on the horizontal axis:

$$
x \in \mathcal{P}_{1} \mathcal{G}_{j} \Leftrightarrow \exists y:(x, y) \in \mathcal{G}_{j} .
$$

We consider the open sets

$$
\mathcal{P}_{1} \mathcal{G}_{j}^{-}=\left\{x \in \mathcal{P}_{1} \mathcal{G}_{j}: x<1\right\}, \quad \mathcal{P}_{1} \mathcal{G}_{j}^{+}=\left\{x \in \mathcal{P}_{1} \mathcal{G}_{j}: x>1\right\} .
$$

We want to compute the degree of the function $f:] 0,+\infty[\rightarrow \mathbb{R}$, defined by

$$
f\left(u_{0}\right)=\dot{\rho}\left(\frac{T}{2} ; u_{0}, 0\right),
$$

on those sets $\mathcal{P}_{1} \mathcal{G}_{j}^{-}$and $\mathcal{P}_{1} \mathcal{G}_{j}^{+}$. Notice that $f\left(u_{0}\right)=F_{1}\left(u_{0}, 0\right)$, where $F$ is the function defined in (18).

In order to compute the degrees $d_{B}\left(f, \mathcal{P}_{1} \mathcal{G}_{j}^{ \pm}\right)$, one can use an argument similar to the one developed in the proof of Lemma 4. Let us summarize it briefly. The sets $\mathcal{P}_{1} \mathcal{G}_{j}^{-}$and $\mathcal{P}_{1} \mathcal{G}_{j}^{+}$are the finite or at most countable union of open intervals. However, the computation of the degree can be reduced to the case when there are only a finite number of them, and the function $f$ changes sign at their end points. In this situation, since $f\left(u_{0}\right)=F_{1}\left(u_{0}, 0\right)$, the same argument used to compute the degree of $F$ in Lemma 4 shows that the number $m_{j}$ of such intervals must be odd, and one proves that

$$
d_{B}\left(f, \mathcal{P}_{1} \mathcal{G}_{j}^{-}\right)=d_{B}\left(f, \mathcal{P}_{1} \mathcal{G}_{j}^{+}\right)=(-1)^{j} .
$$


It is readily seen that the sets $\Omega_{j}^{ \pm}$and $\mathcal{P}_{1} \mathcal{G}_{j}^{ \pm}$have a common core with respect to the Neumann problem $\left(\hat{P}_{0}\right)$, cf. [6]. This means that

(i) $L \rho \neq \hat{N}(\rho, 0)$, for every $\rho \in \partial \Omega_{j}^{ \pm}$;

(ii) $f\left(u_{0}\right) \neq 0$ for every $u_{0} \in \partial \mathcal{P}_{1} \mathcal{G}_{j}^{ \pm}$;

(iii) the set of those $u_{0} \in \mathcal{P}_{1} \mathcal{G}_{j}^{ \pm}$such that $f\left(u_{0}\right)=0$ coincides with the set of initial values $\rho(0)$ of the functions $\rho \in \Omega_{j}^{ \pm}$which are solutions of $L \rho=\hat{N}(\rho, 0)$.

Hence, using [6, Theorem 3] (see also [5], for the periodic problem), the following equalities hold true:

$$
\begin{aligned}
& D_{L}\left(L-\hat{N}(\cdot, 0), \Omega_{j}^{-}\right)=d_{B}\left(f, \mathcal{P}_{1} \mathcal{G}_{j}^{-}\right), \\
& D_{L}\left(L-\hat{N}(\cdot, 0), \Omega_{j}^{+}\right)=d_{B}\left(f, \mathcal{P}_{1} \mathcal{G}_{j}^{+}\right) .
\end{aligned}
$$

The proof is thus completed.

Consider the set $\mathcal{O}_{j}$, introduced in (22), and define

$$
\mathcal{O}_{j}^{-}=\left\{(\rho, \lambda) \in \mathcal{O}_{j}: \rho(0)<\frac{1}{d_{2}}\right\}, \quad \mathcal{O}_{j}^{+}=\left\{(\rho, \lambda) \in \mathcal{O}_{j}: \rho(0)>d_{2}\right\} .
$$

These are open and bounded sets in $X \times[0,1]$. Let us denote by $\overline{\mathcal{O}}_{j}^{-}$and $\partial \mathcal{O}_{j}^{-}$the closure and the boundary of $\mathcal{O}_{j}^{-}$in $X \times[0,1]$, and similarly for $\mathcal{O}_{j}^{+}$. Notice that $\overline{\mathcal{O}}_{j}^{-}$and $\overline{\mathcal{O}}_{j}^{+}$are contained in $X_{+} \times[0,1]$.

Let us fix an integer $J>\max \left\{\kappa_{1}, T / \bar{\tau}\right\}$, where $\kappa_{1}$ is given by Lemma 6 and $\bar{\tau}$ is defined in (23). We now concentrate on the set $\mathcal{O}_{j}^{-}$, i.e.,

$$
\begin{aligned}
\mathcal{O}_{j}^{-}= & \left\{(\rho, \lambda) \in X_{+} \times[0,1]: \kappa(\rho, \lambda) \in\right] j-\frac{1}{4}, j+\frac{1}{4}\left[, \rho(0)<\frac{1}{d_{2}},\right. \\
& \text { and } \left.\mathcal{N}(\rho(t), \dot{\rho}(t))<C_{j}, \text { for every } t \in\left[0, \frac{T}{2}\right]\right\} .
\end{aligned}
$$

Lemma 11 If $j \geq J$ and $\rho_{\lambda}$ solves the Neumann problem $\left(\hat{P}_{\lambda}\right)$, with $\lambda \in[0,1]$ and $\left(\rho_{\lambda}, \lambda\right) \in$ $\overline{\mathcal{O}}_{j}^{-}$, then $\left(\rho_{\lambda}, \lambda\right) \in \mathcal{O}_{j}^{-}$.

Proof If $\left(\rho_{\lambda}, \lambda\right) \in \overline{\mathcal{O}}_{j}^{-}$, we have that $\kappa\left(\rho_{\lambda}, \lambda\right) \in\left[j-\frac{1}{4}, j+\frac{1}{4}\right], \rho_{\lambda}(0) \leq \frac{1}{d_{2}}$, and $\mathcal{N}\left(\rho_{\lambda}(t), \dot{\rho}_{\lambda}(t)\right) \leq C_{j}$, for every $t \in\left[0, \frac{T}{2}\right]$. Being $\rho_{\lambda}$ a solution of $\left(\hat{P}_{\lambda}\right)$, by the choice of $d_{2}$ we know that $\kappa\left(\rho_{\lambda}, \lambda\right)$ is an integer which counts the number of half-rotations around $(1,0)$ of $\left(\rho_{\lambda}(t), \dot{\rho}_{\lambda}(t)\right)$, in the phase plane, as $t$ varies from 0 to $\frac{T}{2}$. Hence, it has to be $\kappa\left(\rho_{\lambda}, \lambda\right)=j$. By Lemma 7 , it has to be $\mathcal{N}\left(\rho_{\lambda}(t), \dot{\rho}_{\lambda}(t)\right)<C_{j}$, for every $t \in\left[0, \frac{T}{2}\right]$. Since $j \geq J>\kappa_{1}$, by Lemma 6 , it has to be $\mathcal{N}\left(\rho_{\lambda}(0), \dot{\rho}_{\lambda}(0)\right)>d_{2} \sqrt{2}$, hence, since $\dot{\rho}_{\lambda}(0)=0$, we have $\rho_{\lambda}(0)<\frac{1}{d_{2}}$. We have thus proved that $\left(\rho_{\lambda}, \lambda\right) \in \mathcal{O}_{j}^{-}$.

So, we have that $0 \notin\left(I-(L-\sigma I)^{-1}(\hat{N}-\sigma I)\right)\left(\partial \mathcal{O}_{j}^{-}\right)$, for every $j \geq J$. By [35, Lemma 1.8], denoting by $\mathcal{O}_{j}^{\lambda,-}$ the set of those $\rho$ such that $(\rho, \lambda) \in \mathcal{O}_{j}^{-}$, we have that $D_{L}\left(L-\hat{N}(\cdot, \lambda), \mathcal{O}_{j}^{\lambda,-}\right)$ is independent of $\lambda \in[0,1]$. Hence,

$$
D_{L}\left(L-\hat{N}(\cdot, 1), \mathcal{O}_{j}^{1,-}\right)=D_{L}\left(L-\hat{N}(\cdot, 0), \mathcal{O}_{j}^{0,-}\right) .
$$


Lemma 12 For every $j \geq J$,

$$
D_{L}\left(L-\hat{N}(\cdot, 0), \mathcal{O}_{j}^{0,-}\right)=(-1)^{j} .
$$

Proof We claim that, if $\rho$ is a solution of $\left(\hat{P}_{0}\right)$, then, for $j \geq J$,

$$
\rho \in \mathcal{O}_{j}^{0,-} \Leftrightarrow \rho \in \Omega_{j}^{-} \text {. }
$$

Indeed, if $\rho \in \mathcal{O}_{j}^{0,-}$, since it solves the Neumann problem and $\rho(0)<\frac{1}{d_{2}}$, it has to be $\kappa(\rho, 0)=j$. Recall that a solution of $\left(\hat{P}_{0}\right)$ can be naturally extended to a $T$-periodic and even function. Hence, extending $\rho$ in such a way, it has minimal period $\frac{T}{j}$, so that the orbit $(\rho(t), \dot{\rho}(t))$ belongs to $\mathcal{G}_{j}$, for every $t$. This means that $\rho \in \Omega_{j}^{-}$.

On the other hand, if $\rho \in \Omega_{j}$, then the periodic extension of $\rho$ has minimal period $\frac{T}{j}$. Since $j \geq J>T / \bar{\tau}$, we have that $\rho(0)<\frac{1}{d_{2}}$, hence $(\rho(t)-1)^{2}+\dot{\rho}^{2}(t)>\frac{1}{4}$ for every $t$, so that it has to be $\kappa(\rho, 0)=j$. By Lemma $7, \mathcal{N}\left(\rho_{\lambda}(t), \dot{\rho}_{\lambda}(t)\right)<C_{j}$, for every $t \in\left[0, \frac{T}{2}\right]$. So, $\rho \in \mathcal{O}_{j}^{0,-}$.

Having proved this, we then have that

$$
D_{L}\left(L-\hat{N}(\cdot, 0), \mathcal{O}_{j}^{0,-}\right)=D_{L}\left(L-\hat{N}(\cdot, 0), \Omega_{j}^{-}\right) .
$$

The conclusion follows, by Lemma 10 . that

An analogous argument can be used for $\mathcal{O}_{j}^{+}$, so we can conclude, using the same notations,

$$
D_{L}\left(L-\hat{N}(\cdot, 1), \mathcal{O}_{j}^{1,-}\right) \neq 0, \quad D_{L}\left(L-\hat{N}(\cdot, 1), \mathcal{O}_{j}^{1,+}\right) \neq 0 .
$$

The proof of Theorem 2 now continues following the lines of the proof of Theorem 1, thus obtaining two branches of solutions for (1) with small angular momentum, one coming from $\mathcal{O}_{j}^{1,-}$ and the other one coming from $\mathcal{O}_{j}^{1,+}$.

We conclude this section by considering the general situation, where the functions $g$ and $e$ are defined on an interval $] a, b$, with $0 \leq a<b \leq+\infty$, with $g$ being "superlinear" both in $a$ and in $b$, and $e$ being bounded. We already analysed the case $a=0, b=+\infty$. The assumptions at $a$ are now the following:

$$
\begin{aligned}
& \left(\mathrm{H} 1^{\prime}\right) \lim _{r \rightarrow a^{+}} g(r)=-\infty, \\
& \left(\mathrm{H} 2^{\prime}\right) \lim _{r \rightarrow a^{+}} G(r)=+\infty,
\end{aligned}
$$

where $G:] a, b[\rightarrow \mathbb{R}$ is a primitive of $g$. As shown in [17], the same type of behaviour of the solutions $\rho(t)$ of the radial equation, as described in the previous section, is met when the superlinear assumption (H3) is replaced, when $b<+\infty$, by the two assumptions

$$
\begin{gathered}
\left(\mathrm{H}^{\prime}\right) \lim _{r \rightarrow b^{-}} g(r)=+\infty, \\
\left(\mathrm{H}^{\prime \prime}\right) \lim _{r \rightarrow b^{-}} G(r)=+\infty .
\end{gathered}
$$

We can then state the following general result.

Theorem 3 Assume $\left(\mathrm{H}^{\prime}\right)$, ( $\left(\mathrm{H} 2^{\prime}\right)$, and, either $(\mathrm{H} 3)$ if $b=+\infty$, or $\left(\mathrm{H}^{\prime}\right)$ and $\left(\mathrm{H}^{\prime \prime}\right)$ if $b<$ $+\infty$. Concerning the function $e(t, r)$, assume $(\mathrm{H} 4)$ and $(\mathrm{H} 5)$. Let $\bar{r}$ be a fixed number in 
] $a, b\left[\right.$. Then, for every integer $N \geq 1$ there exists a $k_{N} \geq 1$ such that, for every integer $k \geq k_{N}$, system (1) has at least $2 N$ periodic solutions $x_{k, 1}^{(i)}(t), x_{k, 2}^{(i)}(t), \ldots, x_{k, N}^{(i)}(t)$, with $i \in\{1,2\}$, having minimal period $k T$, which make exactly one revolution around the origin in the period time $k T$. Moreover, these solutions have the following properties:

(i) the function $\left|x_{k, j}^{(i)}(t)\right|$ is T-periodic and even;

(ii) there is an integer $J \geq 1$ such that the equality $\left|x_{k, j}^{(i)}(t)\right|=\bar{r}$ holds for exactly $J+j$ values of $t$ in $\left[0, \frac{T}{2}[\right.$;

(iii) there are two constants $\tilde{c}_{N}, \hat{c}_{N}$ such that

$$
a<\tilde{c}_{N} \leq\left|x_{k, j}^{(i)}(t)\right| \leq \hat{c}_{N}<b,
$$

for every $t \in \mathbb{R}$, every $j=1,2, \ldots, N$, and every $k \geq k_{N}$;

(iv) if $\mu_{k, j}^{(i)}$ denotes the angular momentum associated with $x_{k, j}^{(i)}(t)$, then

$$
\lim _{k \rightarrow \infty} \mu_{k, j}^{(i)}=0
$$

(v) one has

$$
\left|x_{k, j}^{(1)}(0)\right|<\bar{r}, \quad\left|x_{k, j}^{(2)}(0)\right|>\bar{r} .
$$

Proof We just give a sketch, since, in view of [17], the arguments are completely analogous to the ones in the proofs of Theorems 1 and 2. In the phase plane, we work in the set

$$
\mathcal{S}=\left\{(u, v) \in \mathbb{R}^{2}: a<u<b\right\},
$$

and we introduce the function $\mathcal{N}: \mathcal{S} \rightarrow \mathbb{R}$, defined by

$$
\mathcal{N}(u, v)= \begin{cases}\left(\frac{1}{(u-a)^{2}}+u^{2}+v^{2}\right)^{\frac{1}{2}} & \text { if } b=+\infty, \\ \left(\frac{1}{(u-a)^{2}}+\frac{1}{(b-u)^{2}}+v^{2}\right)^{\frac{1}{2}} & \text { if } b<+\infty .\end{cases}
$$

Lemmas 1 and 2 then hold exactly the same.

Recall that, in the case $a=0, b=+\infty$, we have chosen $\bar{r}=1$, for simplicity. This will not be the case now (unless we perform a change of variable, so to have that $a<1<b$ ). Moreover, we have often made use of intervals of the type $I_{d}=\left[\frac{1}{d}, d\right]$, for some $d>1$, which now need to be replaced by $I_{d}=\left[a+\frac{1}{d}, d\right]$, if $b=+\infty$, and by $I_{d}=\left[a+\frac{1}{d}, b-\frac{1}{d}\right]$, if $b<+\infty$, with $d>1$ such that $\bar{r} \in I_{d}$. Taking into account these modifications, Lemma 3 holds, where $(a)$ and $(b)$ now read as

$$
\begin{aligned}
& \text { (a) } \lim _{u_{0} \rightarrow a^{+}} \mathcal{N}\left(\rho_{\lambda}\left(t ; u_{0}, 0\right), \dot{\rho}_{\lambda}\left(t ; u_{0}, 0\right)\right)=\lim _{u_{0} \rightarrow b^{-}} \mathcal{N}\left(\rho_{\lambda}\left(t ; u_{0}, 0\right), \dot{\rho}_{\lambda}\left(t ; u_{0}, 0\right)\right)=+\infty, \\
& \quad \text { uniformly in } t \in\left[0, \frac{T}{2}\right] ; \\
& \text { (b) } \lim _{u_{0} \rightarrow a^{+}} \theta_{\lambda}\left(\frac{T}{2} ; u_{0}, 0\right)=\lim _{u_{0} \rightarrow b^{-}} \theta_{\lambda}\left(\frac{T}{2} ; u_{0}, 0\right)=-\infty .
\end{aligned}
$$

The sets $\mathcal{G}_{j}$ are defined as in (17), and Lemma 4 is proved exactly as before.

The set $X_{+}$will now be defined as

$$
X_{+}=\{\rho \in X: a<\min \rho \leq \max \rho<b\},
$$

so that, again,

$$
\Omega_{j}=\left\{\rho \in X_{+}:(\rho(t), \dot{\rho}(t)) \in \mathcal{G}_{j}, \text { for every } t \in\left[0, \frac{T}{2}\right]\right\},
$$


and we define the sets

$$
\Omega_{j}^{-}=\left\{\rho \in \Omega_{j}: \rho(0)<\bar{r}\right\}, \quad \Omega_{j}^{+}=\left\{\rho \in \Omega_{j}: \rho(0)>\bar{r}\right\} .
$$

Lemma 10 is now proved as above. We define the function $\kappa: X_{+} \times[0,1] \rightarrow \mathbb{R}$ as

$$
\kappa(\rho, \lambda)=\frac{1}{\pi} \int_{0}^{T / 2} \frac{\dot{\rho}^{2}(t)+(g(\rho(t))-\lambda e(t, \rho(t)))(\rho(t)-\bar{r})}{\max \left\{(\rho(t)-\bar{r})^{2}+\dot{\rho}^{2}(t), \frac{1}{4} \min \{\bar{r}-a, b-\bar{r}\}\right\}} \mathrm{d} t .
$$

The two implications (20) and (21) now read as

$$
u \notin \stackrel{\circ}{I}_{d_{2}} \Rightarrow \mathcal{N}(u, 0) \geq d_{2},
$$

and

$$
\mathcal{N}(u, 0)>d_{2} \sqrt{2} \Rightarrow u \notin I_{d_{2}},
$$

respectively. Lemmas 6 and 7 then hold.

Define the sets

$$
\begin{gathered}
\mathcal{O}_{j}^{-}=\left\{(\rho, \lambda) \in X_{+} \times[0,1]: \kappa(\rho, \lambda) \in\right] j-\frac{1}{4}, j+\frac{1}{4}\left[, \rho(0)<\min I_{d_{2}},\right. \\
\text { and } \left.\mathcal{N}(\rho(t), \dot{\rho}(t))<C_{j}, \text { for every } t \in\left[0, \frac{T}{2}\right]\right\}, \\
\mathcal{O}_{j}^{+}=\left\{(\rho, \lambda) \in X_{+} \times[0,1]: \kappa(\rho, \lambda) \in\right] j-\frac{1}{4}, j+\frac{1}{4}\left[, \rho(0)>\max I_{d_{2}},\right. \\
\text { and } \left.\mathcal{N}(\rho(t), \dot{\rho}(t))<C_{j}, \text { for every } t \in\left[0, \frac{T}{2}\right]\right\} .
\end{gathered}
$$

and denote by $\mathcal{O}_{j}^{\lambda, \pm}$ the set of those $\rho$ such that $(\rho, \lambda) \in \mathcal{O}_{j}^{ \pm}$. Lemmas 11 and 12 then hold, yielding

$$
D_{L}\left(L-\hat{N}(\cdot, 1), \mathcal{O}_{j}^{1,-}\right) \neq 0, \quad D_{L}\left(L-\hat{N}(\cdot, 1), \mathcal{O}_{j}^{1,+}\right) \neq 0 .
$$

The proof is concluded following the lines of the proof of Theorem 1, thus obtaining, like in Theorem 2, two branches of solutions for (1), with small angular momentum.

Remark 2 Notice that, in particular, when $0<a<b<+\infty$, we are in the situation of an annular potential well. The existence of periodic solutions in the case of a potential well for more general domains in $\mathbb{R}^{N}$ has been proved by the use of variational methods by several authors, see, e.g. [3,11,15].

\section{References}

1. Ambrosetti, A., Coti Zelati, V.: Periodic Solutions of Singular Lagrangian Systems, Progress in Nonlinear Differential Equations and their Applications, vol 10. Birkhäuser, Boston (1993)

2. Arnold, V.I.: Mathematical Methods of Classical Mechanics, Grad. Texts in Math., vol. 60. 2nd edn. Springer, Heidelberg (1978)

3. Benci, V.: Normal modes of a Lagrangian system constrained in a potential well. Ann. Inst. H. Poincaré 1, 379-400 (1984)

4. Capietto, A., Henrard, M., Mawhin, J., Zanolin, F.: A continuation approach to some forced superlinear Sturm-Liouville boundary value problems. Topol. Meth. Nonlin. Anal. 3, 81-100 (1994)

5. Capietto, A., Mawhin, J., Zanolin, F.: A continuation approach to superlinear periodic boundary value problems. J. Differ. Equ. 88, 347-395 (1990)

6. Capietto, A., Mawhin, J., Zanolin, F.: A duality theorem for coincidence equations with applications to superlinear Neumann boundary value problems. J. Niger. Math. Soc. 11, 83-105 (1992) 
7. Capietto, A., Mawhin, J., Zanolin, F.: On the existence of two solutions with a prescribed number of zeros for a superlinear two-point boundary value problem. Topol. Meth. Nonlin. Anal. 6, 175-188 (1995)

8. Capozzi, A., Greco, C., Salvatore, A.: Lagrangian systems in the presence of singularities. Proc. Amer. Math. Soc. 102, 125130 (1988)

9. Chu, J., Franco, D.: Non-collision periodic solutions of second order singular dynamical systems. J. Math. Anal. Appl. 344, 898-905 (2008)

10. Coti Zelati, V.: Dynamical systems with effective-like potential. Nonlinear Anal. 12, 209-222 (1988)

11. Coti Zelati, V., Li, S., Wu, S.: Periodic solutions for a class of singular nonautonomous second order systems in a potential well. In: Variational Methods in Nonlinear Analysis. Gordon and Breach, Basel (1995)

12. Degiovanni, M., Giannoni, F., Marino, A.: Dynamical systems with Newtonian type potentials, Atti Accad. Naz. Lincei Rend. Cl. Sci. Fis. Mat. Natur. (8) 81 (1987) (n.3, 271-277, 1988)

13. Del Pino, M., Manásevich, R.: Infinitely many $T$-periodic solutions for a problem arising in nonlinear elasticity. J. Differ. Equ. 103, 260-277 (1993)

14. Ding, T., Zanolin, F.: Periodic solutions of Duffing's equations with superquadratic potential. J. Differ. Equ. 97, 328-378 (1992)

15. Ding, Y., Li, S.: Periodic solutions of some singular dynamical systems in a potential well. Chin. J. Contemp. Math. 13(1992), 299-307 (1993)

16. Fonda, A.: Periodic solutions for a conservative system of differential equations with a singularity of repulsive type. Nonlinear Anal. 24, 667-676 (1995)

17. Fonda, A., Manásevich, R., Zanolin, F.: Subharmonic solutions for some second-order differential equations with singularities. SIAM J. Math. Anal. 24, 1294-1311 (1993)

18. Fonda, A., Toader, R.: Periodic orbits of radially symmetric Keplerian-like systems: a topological degree approach. J. Differ. Equ. 244, 3235-3264 (2008)

19. Fonda, A., Toader, R.: Periodic orbits of radially symmetric systems with a singularity: the repulsive case. Adv. Nonlinear Stud. (to appear)

20. Fonda, A., Toader, R.: Periodic solutions of radially symmetric perturbations of Newtonian systems, preprint. Available on-line at www.dmi.units.it/pubblicazioni/Quaderni_Matematici/598_2009.pdf

21. Fonda, A., Toader, R.: Radially symmetric systems with a singularity and asymptotically linear growth. Nonlinear Anal. (to appear)

22. Fonda, A., Ureña, A.: Periodic, subharmonic, and quasi-periodic oscillations under the action of a central force. Discret. Cont. Dynam. Syst. 29, 169-192 (2011)

23. García-Huidobro, M., Manásevich, R., Zanolin, F.: Strongly nonlinear second-order ODEs with rapidly growing terms. J. Math. Anal. Appl. 202, 1-26 (1996)

24. Gaines, E., Mawhin, J.: Coincidence Degree and Nonlinear Differential Equations. Lecture Notes in Math, vol. 568. Springer, Berlin (1977)

25. Gordon, W.B.: Conservative dynamical systems involving strong forces. Trans. Am. Math. Soc. 204, 113-135 (1975)

26. Habets, P., Sanchez, L.: Periodic solutions of dissipative dynamical systems with singular potentials. Differ. Integr. Equ. 3, 1139-1149 (1990)

27. Jacobowitz, H.: Periodic solutions of $x^{\prime \prime}+f(x, t)=0$ via the Poincaré-Birkhoff theorem. J. Differ. Equ. 20, 37-52 (1976)

28. Krasnosel'skii, M.A., Zabreiko, P.P.: Geometrical Methods of Nonlinear Analysis. Springer, Berlin (1984)

29. Lazer, A.C., Solimini, S.: On periodic solutions of nonlinear differential equations with singularities. Proc. Am. Math. Soc. 99, 109-114 (1987)

30. Levi, M.: Quasiperiodic motions in superquadratic time-periodic potentials. Comm. Math. Phys. 143, 43-83 (1991)

31. Llibre, J., Ortega, R.: On the families of periodic orbits of the Sitnikov problem. SIAM J. Appl. Dyn. Syst. 7, 561-576 (2008)

32. Mawhin, J.: Leray-Schauder degree: a half century of extensions and applications. Topol. Meth. Nonlin. Anal. 14, 195-228 (1999)

33. Morris, G.R.: An infinite class of periodic solutions of $\ddot{x}+2 x^{3}=p(t)$. Proc. Cambridge Philos. Soc. 61, 157-164 (1965)

34. van Noort, M., Porter, M.A., Yi, Y., Chow, S.-N.: Quasiperiodic dynamics in Bose-Einstein condensates in periodic lattices and superlattices. J. Nonlinear Sci. 17, 59-83 (2007)

35. Rabinowitz, P.H.: Some aspects of nonlinear eigenvalue problems. Rocky Mount. J. Math. 3, 161-202 (1973)

36. Rabinowitz, P.H.: Variational methods for Hamiltonian systems. In: Handbook of Dynamical Systems, vol. 1A pp. 1091-1127. North-Holland, Amsterdam (2002) 
37. Serra, E., Terracini, S.: Noncollision solutions to some singular minimization problems with Keplerianlike potentials. Nonlinear Anal. 22, 45-62 (1994)

38. Solimini, S.: On forced dynamical systems with a singularity of repulsive type. Nonlinear Anal. 14, 489-500 (1990)

39. Torres, P.J.: Non-collision periodic solutions of forced dynamical systems with weak singularities. Discret. Contin. Dyn. Syst. 11, 693-698 (2004)

40. Zeidler, E.: Nonlinear Functional Analysis and its Applications, vol. 1. Springer, New YorkHeidelberg (1986)

41. Zhong-Heng, G., Solecki, R.: Free and forced finite amplitude oscillations of an elastic thick walled hollow sphere made in incompressible material. Arch. Mech. Stos. 25, 427-433 (1963) 